\title{
Supporting Information: Microscopic Theory of Plasmons in Substrate-supported Borophene
}

\author{
Anubhab Haldar, ${ }^{\dagger}$ Cristian L. Cortes, ${ }^{\ddagger}$ Pierre Darancet, ${ }^{*}, \uparrow, \uparrow$ and Sahar \\ Sharifzadeh*, $\S, \|$ \\ $\dagger$ Department of Electrical and Computer Engineering, Boston University, Boston, \\ Massachusetts 02215, United States \\ $\ddagger$ Center for Nanoscale Materials, Argonne National Laboratory, Argonne, IL 60439, USA \\ \Northwestern Argonne Institute of Science and Engineering, Evanston, IL 60208, USA \\ $\S$ Division of Materials Science and Engineering, Boston University, Boston, MA 02215, \\ USA \\ ||Department of Electrical and Computer Engineering, Boston University, Boston, MA \\ 02215, USA
}

E-mail: pdarancet@anl.gov; ssharifz@bu.edu 


\section{Contents}

1 Computational details $\quad 4$

1.1 Borophene and borophene $/ \operatorname{Ag}(111) \ldots \ldots \ldots \ldots$

1.2 Borophene/Al(111) . . . . . . . . . . . . . . . 6

2 Coupled polarizability model fitting procedure $r$

3 Definitions of computed quantities $\quad 9$

4 Models of interacting polarizabilities $\quad 10$

4.1 Time-dependent DFT . . . . . . . . . . . . . . . . . . . 10

4.2 Coupled polarizability model . . . . . . . . . . . . . . . . . . . . 12

4.3 Coupled induced density model . . . . . . . . . . . . . . . . . 14

4.4 Derivation of coupled polarizabilities in the projector formalism . . . . . . . 14

4.5 Local expression of plasmon resonances in the quasistatic regime . . . . . . 17

5 Derivation of the supported polarizabilities $\quad 19$

6 Independent-particle polarizabilities 20

7 Conventional bandpath $\quad 22$

8 Bandpaths for polarizability 'wedges' 23

9 Charge transfer at the borophene-Ag(111) interface 25

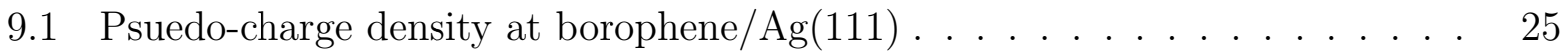

9.2 Bader analysis of charge transfer between borophene and substrates . . . . $\quad 25$

10 Influence of a van der Waals correction to geometry on the predicted $\begin{array}{ll}\text { borophene response } & 28\end{array}$ 
11 Borophene/Ag(111) model fits $\quad 28$

11.1 Borophene $/ \mathrm{Ag}(111) \ldots \ldots \ldots \ldots$

12 Model robustness $\quad 39$

12.1 Borophene/Ag(111): model sensitivity to $\mathrm{t}(\mathbf{q}) \ldots \ldots$. . . . . . . . . 40

12.2 Borophene/Al(111): model sensitivity to $\mathrm{t}(\mathbf{q}) \ldots \ldots$. . . . . . . . 41

12.3 Sensitivity to surface corrugation . . . . . . . . . . . . . . . . 42

13 Model transferability $\quad 43$

13.1 Model-Predicted polarizability for borophene/Al(111) compared with fit . . . 43

13.2 Distance-dependence of the coupling parameter . . . . . . . . . . . 45

14 Convergence $\quad 47$

$\begin{array}{ll}15 \text { Scripts } & 48\end{array}$

$\begin{array}{ll}\text { References } & 49\end{array}$ 


\section{Computational details}

All calculations were performed with the help of the Atomic Simulation Environment. ${ }^{1}$ We perform first-principles DFT and linear response TDDFT calculations on a $2 \times 1$ supercell of the isolated and $\operatorname{Ag}(111)$ substrate-supported $\beta_{12}$ borophene allotrope. We use a $2 \times 2$ supercell of 6-layer $\operatorname{Ag}(111)$, which is commensurate with the borophene supercell such that $<1 \%$ strain was introduced by formation of the interface.

For the three elemental metals studied in Figure 3 of the main text $(\mathrm{Ag}, \mathrm{Au}, \mathrm{Al})$, we calculate the dielectric response of the fcc(111) 6-layer surface. For TiN, a 4-layer (100) surface using the conventional unit cell of the cubic crystal, obtained from the Materials Project $^{2}$ (id: mp-492) database was used. The resulting polarizabilities were used as input for the electrodynamic model.

For the two subsections below that show calculations using LDA, the versions of $\mathrm{PBE}^{3}$ with a DFT-D3 (Becke-Johnson damping) ${ }^{4}$ correction are identical except for the change in the functional, and addition of the van der Waals description.

\section{$1.1 \quad$ Borophene and borophene/Ag(111)}

The geometry was optimized within the local density approximation (LDA) 5,6 using the VASP package. ${ }^{7,8}$ PAW potentials ${ }^{9,10}$ were used to represent the core and nuclei of atoms with a planewave cutoff of $450 \mathrm{eV}$ with a Monkhorst-Pack k-point mesh of $24 \times 28 \times 1$ for Brillouin zone integration. Forces were converged to under $0.1 \mathrm{meV} / \AA$ and a MethfesselPaxton ${ }^{11}$ smearing of order 1 and magnitude $0.2 \mathrm{eV}$ was used to describe the metallic nature of borophene. For borophene on $\mathrm{Ag}(111)$, a Gaussian smearing of $0.2 \mathrm{eV}$ was used to described the metallic nature of the system. For freestanding borophene, the unit cell and the full stress tensor including off-diagonal elements, was relaxed, keeping the volume of the simulation cell constant. For borophene on silver, 6 layers of silver were considered, with the atomic positions of the bottom two layers fixed to mimic the bulk. The borophene 
atoms, as well as the top 4 layers of silver atoms were optimized using the conjugate-gradient algorithm as implemented in VASP.

The optimized $2 \times 1$ borophene lattice constants were $\mathrm{a}=5.805 \AA$ along the $\mathrm{x}$ direction, and $\mathrm{b}=5.015 \AA$ along the $\mathrm{y}$ direction. The magnitude of the $\mathrm{z}$ dimension of the simulation cell was approximately $13.7 \AA$ to suppress interactions between artificial periodic images. For borophene on silver in the $\mathrm{v}_{1 / 6}-30^{\circ}$ phase of reference, ${ }^{12}$ the borophene was strained to lattice constants of $5.784 \AA$ along the $\mathrm{x}$ direction and $5.009 \AA$ along the $\mathrm{y}$ direction to be commensurate with the silver lattice. This corresponds to a strain of $0.4 \%$ along the $\mathrm{x}$ direction and $0.1 \%$ along the y direction.

Linear response calculations including intraband transitions were computed within the random-phase approximation (RPA) both including and excluding local field effects, with the DFT-GLLBSC functional, ${ }^{13}$ as implemented in GPAW v. $1.5 .2 .{ }^{14-16}$ We apply a $2 \mathrm{D}$ Coulomb truncation to avoid spurious interactions along the $\mathrm{z}$ axis and ensured that the length of the z-direction encompasses the induced densities. The magnitude of the $\mathrm{z}$ dimension was approximately $11 \AA, 36.8 \AA, 46.8 \AA, 56.8 \AA, 66.8 \AA$ for borophene and borophene/ $\mathrm{Ag}(111)$ at substrate-2D distances of $2 \AA, 7 \AA, 12 \AA$, and $17 \AA$ above equilibrium, respectively. The core and nuclei were described with PAW potentials of GPAW version 0.9.2. A planewave cutoff of $350 \mathrm{eV}$ was used, which was sufficient to converge the absorption and polarizability spectra. A Fermi-Dirac smearing of $1 \mathrm{meV}$ for freestanding borophene and $50 \mathrm{meV}$ for borophene/ $\mathrm{Ag}(111)$ was used.

A k-point mesh of $24 \times 28 \times 1$ was used for the ground state calculation, and $120 \times 140 \times 1$ were used for the linear response calculations of freestanding borophene. For borophene on $\operatorname{Ag}(111)$, we utilized an anisotropic k-point mesh for the linear response calculation in order to allow a finer k-point mesh to be used in the direction of interest for the polarizability calculations. The k-point mesh was $72 \times 28 \times 1$ for momentum along the $\mathrm{x}$-direction and $24 \times 84 \times 1$ along the $\mathrm{y}$-direction, allowing a finer momentum resolution (which must be commensurate with the k-point mesh of the ground state calculation). The dense k-point 
mesh leads to smooth linear response spectra. We compared the absorption and polrizability spectra predicted using this k-point mesh compared with an isotropic one for borophene and $\operatorname{Ag}(111)$ separately, and the interface, and determined that the difference in the calculated EELS spectrum is negligible within the energy window of interest. The dielectric matrix elements were expanded in a planewave basis of $50 \mathrm{eV}$ for freestanding borophene and 25 eV for borophene on silver. 32 and 192 unoccupied bands were used to build the dielectric matrix for borophene and borophene on silver respectively.

The EELS spectrum and the macroscopic polarizability were evaluated on a nonlinear frequency grid ranging from $0-25 \mathrm{eV}$ for borophene (the highest transition energy) $0-15 \mathrm{eV}$ for borophene on $\mathrm{Ag}(111)$. The initial spacing of points in the frequency grid was $0.025 \mathrm{eV}$ for freestanding borophene and $0.05 \mathrm{eV}$ for borophene on silver and increased to twice the initial value at $10 \mathrm{eV}$. Additionally, the Hilbert transform was used to calculate the real part of the dielectric function from the imaginary part, and it was converged with respect to the frequency range.

For the three elemental metals studied in Figure 3 of the main text $(\mathrm{Ag}, \mathrm{Au}, \mathrm{Al})$, we calculate the dielectric response of the fcc(111) 6-layer surface. For TiN, a 4-layer (100) surface using the conventional unit cell of the cubic crystal, obtained from the Materials Project $^{2}$ (id: mp-492) database was used. The resulting polarizabilities were used as input for the electrodynamic model.

\subsection{Borophene/Al(111)}

The geometry was optimized within the local density approximation (LDA) ${ }^{5,6}$ using the VASP package. ${ }^{7,8}$ PAW potentials ${ }^{9,10}$ were used to represent the core and nuclei of atoms with a planewave cutoff of $450 \mathrm{eV}$ with a Monkhorst-Pack k-point mesh of $24 \times 28 \times 1$ for

Brillouin zone integration. For borophene on $\mathrm{Al}(111)$, a Methfessel-Paxton ${ }^{11}$ smearing of order 1 and magnitude $0.1 \mathrm{eV}$ was used to described the metallic nature of the system. Forces were optimized to an energy gradient of $1 \mathrm{meV} / \AA$. 
6 layers of aluminum were considered, with the atomic positions of the bottom two layers fixed to mimic the bulk. The borophene atoms, as well as the top 4 layers of aluminum atoms were optimized using the RMM-DIIS geometry optimization algorithm as implemented in VASP.

For borophene on aluminum in the $\mathrm{v}_{1 / 6}-30^{\circ}$ phase of reference, ${ }^{12}$ the borophene was strained to lattice constants of $5.728 \AA$ along the x direction and $4.960 \AA$ along the y direction to be commensurate with the aluminum lattice. This corresponds to a strain of $1.3 \%$ along the $\mathrm{x}$ direction and $1.1 \%$ along the $\mathrm{y}$ direction.

Linear response calculations including intraband transitions were computed within the random-phase approximation (RPA) both including and excluding local field effects, with the DFT-GLLBSC functional, ${ }^{13}$ as implemented in GPAW v. 1.5.2. ${ }^{14-16}$ We apply a 2D Coulomb truncation to avoid spurious interactions along the $\mathrm{z}$ axis and ensured that the length of the $\mathrm{z}$-direction encompasses the induced densities. The magnitude of the $\mathrm{z}$ dimension was approximately $43.0 \AA, 53.0 \AA, 63.0 \AA, 73.0 \AA$ for borophene/Al(111) at substrate-2D distances of $5.15 \AA, 10.15 \AA, 15.15 \AA$, and $20.15 \AA$ above equilibrium, respectively. The core and nuclei were described with PAW potentials of GPAW version 0.9.2. A planewave cutoff of $350 \mathrm{eV}$ was used, which was sufficient to converge the absorption and polarizability spectra. A Fermi-Dirac smearing of $50 \mathrm{meV}$ for borophene/Al(111) was used.

For borophene on $\mathrm{Al}(111)$, we utilized an anisotropic k-point mesh for the linear response calculation in order to allow a finer k-point mesh to be used in the direction of interest for the polarizability calculations. The k-point mesh was $72 \times 28 \times 1$ for momentum along the x-direction allowing a finer momentum resolution (which must be commensurate with the k-point mesh of the ground state calculation). The dense k-point mesh leads to smooth linear response spectra. The dielectric matrix elements were expanded in a planewave basis of $25 \mathrm{eV}$ for borophene on aluminum. 102 unoccupied bands were used to build the dielectric matrix for borophene on aluminum respectively.

The macroscopic polarizability was evaluated on a nonlinear frequency grid ranging from 
0-29 eV for borophene on $\mathrm{Al}(111)$. The initial spacing of points in the frequency grid was 0.05 $\mathrm{eV}$ for borophene on aluminum and increased to twice the initial value at $10 \mathrm{eV}$. Additionally, the Hilbert transform was used to calculate the real part of the dielectric function from the imaginary part.

\section{Coupled polarizability model fitting procedure}

For fitting the model, cubic splines from the TDDFT data (real and imaginary part of polarizability as a function of energy) were created and fit to the absolute value of the complex polarizability of the borophene/substrate system within the energy range 0-5 eV, divided into 1000 equally spaced sampling points. Fitting to the real or imaginary parts individually leads to only minor changes in the optimal fitting parameter.

To fit the linear response polarizabilities of the metals, we use the trust region reflective ('trf') algorithm ${ }^{17}$ with a smooth approximation of the 11 loss function ('soft_l1'), ${ }^{18}$ and an initial guess of 0.2 for the numerical value of $t(q)$, explicitly factoring out the $\exp \left(-q \times d_{z}\right)$ behaviour, where $\mathrm{q}$ is the transferred momentum, and $\mathrm{d}_{\mathrm{z}}$ is the mean distance between the image plane of the substrate and the borophene nuclei.

The location of the image plane was determined by performing calculations in the presence of a weak external electric field. Weak external electric fields are applied, and the linear

potential far away from the surface of the slab is extrapolated back to the zero-crossing of the linearized potential. 

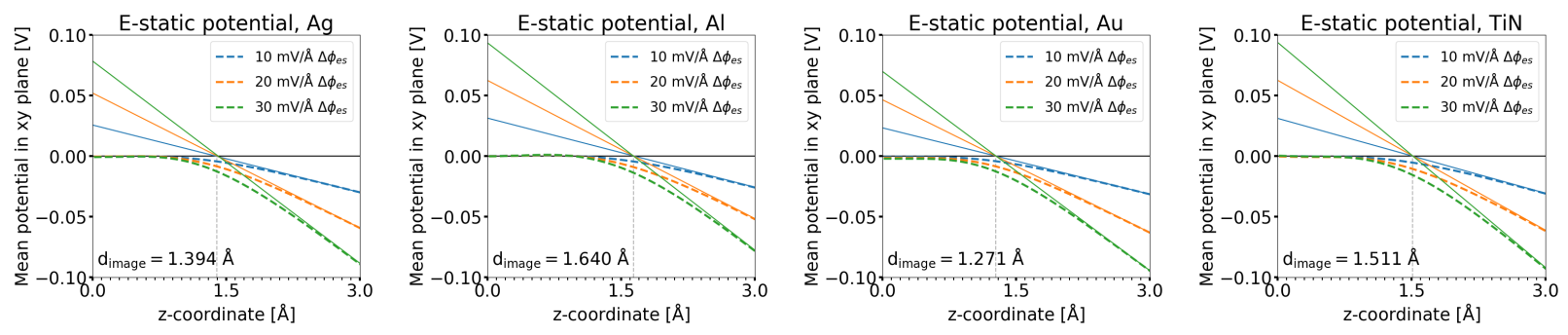

Figure 1: The electrostatic potential near the terminal layer of atoms for slabs of the metals considered (without geometry optimization). The reference z-coordinate origin is the $\mathrm{z}$ coordinate of the terminal atom on the slab.

\section{Definitions of computed quantities}

Representing the noninteracting and Hartree-interacting polarizabilities in a planewave basis set, ${ }^{14}$ we get the following Dyson-like expression relating the two quantities, in the random phase approximation (RPA):

$$
\chi_{\mathbf{G G}^{\prime}}=\chi_{\mathbf{G G}^{\prime}}^{0}+\chi_{\mathbf{G G}_{1}}^{0} \mathrm{v}_{\mathbf{G}_{1} \mathbf{G}_{\mathbf{2}}} \chi_{\mathbf{G}_{2} \mathbf{G}^{\prime}}
$$

where recurring $\mathbf{G}$ indices are summed over.This relates the independent-particle response $\chi_{0}$, to the Hartree-interacting density-density response, $\chi$ and the Coulomb interaction kernel v. The loss spectra defined as

$$
\operatorname{EELS}=-\operatorname{Im}\left(\frac{1}{\varepsilon_{\mathrm{M}}}\right)
$$

However, the macroscopic dielectric function is ill-defined for 2D materials, since the quantity is an average over a 3D unit cell. In 2D, the nonperiodic direction is arbitrary, and in the limit of no interaction with periodic images of the $2 \mathrm{D}$ material (infinite vacuum or a truncated Coulomb kernel), $\varepsilon_{\mathrm{M}}$ is formally 1 in the optical limit. In GPAW, the RPA EELS function including local field effects is calculated using the relation:

$$
\mathrm{EELS}=-\operatorname{Im}(\mathrm{v} \chi[0,0])
$$


where $\chi_{[0,0]}$ is the $\mathbf{G}=\mathbf{G}^{\prime}=0$ element of the interacting density-density response matrix. Although $\varepsilon_{M}$ is ill-defined in lower dimensions, the macroscopic polarizability $\alpha_{\mathrm{M}}$ remains well defined due to the magnitude of the nonperiodic direction(s) being factored out. In low-dimensional materials, the macroscopic polarizability $\alpha_{\mathrm{M}}$ is defined (and implemented in GPAW) by the relation

$$
\alpha \varepsilon_{\mathrm{M}}^{-1}=-\frac{\mathrm{V} \mathrm{v} \chi}{4 \pi}
$$

where $\mathrm{V}$ is determinant of the nonperiodic directions of the simulation cell, $\mathrm{v}$ is the Coulomb

interaction kernel, and $\varepsilon_{\mathrm{M}}^{-1}$ in $2 \mathrm{D}$ is 1 by definition. This relation factors out the dependence on the arbitrary magnitude of the nonperiodic direction, leaving us with a value of $\alpha$ that can be meaningfully converged irrespective of cell volume.

\section{Models of interacting polarizabilities}

\subsection{Time-dependent DFT}

In time-dependent density functional theory, the induced density and self-consistent potential are related by the linear response relation:

$$
\delta \rho(\mathbf{r}, \omega)=\int \mathrm{d} \mathbf{r}^{\prime} \chi_{\mathrm{o}}\left(\mathbf{r}, \mathbf{r}^{\prime}, \omega\right) \varphi_{\text {tot }}\left(\mathbf{r}^{\prime}, \omega\right)
$$

where $\chi_{0}$ is the density-density response function of the non-interacting electron system:

$$
\chi_{\mathrm{o}}\left(\mathbf{r}, \mathbf{r}^{\prime}, \omega\right)=\sum_{\mathrm{k}, \mathrm{k}^{\prime}}\left(\mathrm{f}_{\mathrm{k}}-\mathrm{f}_{\mathrm{k}^{\prime}}\right) \frac{\psi_{\mathrm{k}}(\mathbf{r}) \psi_{\mathrm{k}^{\prime}}^{*}(\mathbf{r}) \psi_{\mathrm{k}}\left(\mathbf{r}^{\prime}\right) \psi_{\mathrm{k}^{\prime}}^{*}\left(\mathbf{r}^{\prime}\right)}{\omega+\varepsilon_{\mathrm{k}}-\varepsilon_{\mathrm{k}^{\prime}}+\mathrm{i} \eta}
$$

The self-consistent potential, $\varphi_{\text {tot }}$, is the sum of the external potential and the induced potential, $\varphi_{\text {tot }}=\varphi_{\text {ext }}+\varphi_{\text {ind }}$, where the induced contribution consists of an electrostatic part as well as the electron-electron exchange and correlation contributions, $\varphi_{\text {ind }}=\varphi_{\text {st }}+\varphi_{\mathrm{xc}}$. 
The electrostatic potential obeys the Poisson equation,

$$
\nabla^{2} \varphi_{\text {st }}(\mathbf{r})=-4 \pi \delta \rho(\mathbf{r})
$$

with a solution that can be written as:

$$
\varphi_{\text {st }}(\mathbf{r})=\int \mathrm{d} \mathbf{r}^{\prime} \mathrm{v}\left(\mathbf{r}, \mathbf{r}^{\prime}\right) \delta \rho\left(\mathbf{r}^{\prime}\right)
$$

where $\mathrm{v}\left(\mathbf{r}, \mathbf{r}^{\prime}\right)$ is the electrostatic Green function due to a single point charge. In free-space, it is given by:

$$
\mathrm{v}\left(\mathbf{r}, \mathbf{r}^{\prime}\right)=\frac{1}{\left|\mathbf{r}-\mathbf{r}^{\prime}\right|}
$$

however this result can be made more general if we have piece-wise background permittivities. Assuming that we can ignore the exchange contributions, we can derive the self-consistent equations for the induced density by combining eqns (5) and (8):

$$
\delta \rho(\mathbf{r}, \omega)=\int \mathrm{d} \mathbf{r}^{\prime} \chi_{0}\left(\mathbf{r}, \mathbf{r}^{\prime}, \omega\right) \varphi_{\operatorname{ext}}\left(\mathbf{r}^{\prime}, \omega\right)+\int \mathrm{d} \mathbf{r}^{\prime} \int \mathrm{d} \mathbf{r}^{\prime \prime} \chi_{\mathrm{o}}\left(\mathbf{r}, \mathbf{r}^{\prime \prime}, \omega\right) \mathrm{v}\left(\mathbf{r}^{\prime \prime}, \mathbf{r}^{\prime}\right) \delta \rho\left(\mathbf{r}^{\prime}, \omega\right)
$$

In operator form, this equation is written as:

$$
\delta \rho=\chi_{0} \varphi_{\text {ext }}+\chi_{0} v \delta \rho
$$

with the solution:

$$
\delta \rho=\left(1-\chi_{\mathrm{ov}}\right)^{-1} \chi_{\mathrm{o}} \varphi_{\mathrm{ext}}
$$

where the new effective susceptibility is defined as:

$$
\chi_{\text {eff }}=\left(1-\chi_{\mathrm{ov}}\right)^{-1} \chi_{\mathrm{o}}
$$


The density-density response function, $\chi_{\mathrm{o}}\left(\mathbf{r}, \mathbf{r}^{\prime}\right)$, is obtained through ab-initio DFT calculations.

\subsection{Coupled polarizability model}

This section derives a coupled mode theory for DFT-derived polarizabilities. We aim to solve the following coupled set of equations for polarization vectors $\mathbf{P}_{1}(\mathbf{r}, \omega)$ and $\mathbf{P}_{2}(\mathbf{r}, \omega)$,

$$
\begin{aligned}
& \mathbf{P}_{1}\left(\mathbf{r}_{1}, \omega\right)=\int \mathrm{d}^{3} \mathbf{r}^{\prime} \chi_{1}\left(\mathbf{r}_{1}, \mathbf{r}^{\prime} ; \omega\right) \mathbf{E}\left(\mathbf{r}^{\prime}, \omega\right), \\
& \mathbf{P}_{2}\left(\mathbf{r}_{2}, \omega\right)=\int \mathrm{d}^{3} \mathbf{r}^{\prime} \chi_{2}\left(\mathbf{r}_{2}, \mathbf{r}^{\prime} ; \omega\right) \mathbf{E}\left(\mathbf{r}^{\prime}, \omega\right),
\end{aligned}
$$

where the electric field at point $\mathbf{r}$ is given by:

$$
\mathbf{E}(\mathbf{r}, \omega)=\mathbf{E}_{\mathrm{o}}(\mathbf{r}, \omega)+\int \mathrm{d}^{3} \mathbf{r}_{1} \mathbf{G}\left(\mathbf{r}, \mathbf{r}_{1} ; \omega\right) \cdot \mathbf{P}_{1}\left(\mathbf{r}_{1}, \omega\right)+\int \mathrm{d}^{3} \mathbf{r}_{2} \mathbf{G}\left(\mathbf{r}, \mathbf{r}_{2} ; \omega\right) \cdot \mathbf{P}_{2}\left(\mathbf{r}_{2}, \omega\right)
$$

To simplify these expressions, we assume $\chi_{i}$ and $\mathbf{G}$ are translational invariant quantities. This assumption allows us to re-write the equations in reciprocal space as:

$$
\begin{aligned}
& \mathbf{P}_{1}(\mathbf{q}, \omega)=\chi_{1}(\mathbf{q} ; \omega) \cdot \mathbf{E}(\mathbf{q}, \omega), \\
& \mathbf{P}_{2}(\mathbf{q}, \omega)=\chi_{2}(\mathbf{q} ; \omega) \cdot \mathbf{E}(\mathbf{q}, \omega),
\end{aligned}
$$

The electric field can also be written as:

$$
\mathbf{E}(\mathbf{q}, \omega)=\mathbf{E}_{\mathrm{o}}(\mathbf{q}, \omega)+\mathbf{G}(\mathbf{q} ; \omega) \cdot \mathbf{P}_{1}(\mathbf{q}, \omega)+\mathbf{G}(\mathbf{q} ; \omega) \cdot \mathbf{P}_{2}(\mathbf{q}, \omega)
$$


Substituting the expression for the total electric field into (29)-(30), we find the following set of coupled equations:

$$
\begin{aligned}
& \mathbf{P}_{1}(\mathbf{q}, \omega)=\chi_{1}(\mathbf{q} ; \omega) \cdot\left[\mathbf{E}_{\mathrm{o}}(\mathbf{q}, \omega)+\mathbf{G}(\mathbf{q} ; \omega) \cdot \mathbf{P}_{1}(\mathbf{q}, \omega)+\mathbf{G}(\mathbf{q} ; \omega) \cdot \mathbf{P}_{2}(\mathbf{q}, \omega)\right], \\
& \mathbf{P}_{2}(\mathbf{q}, \omega)=\chi_{2}(\mathbf{q} ; \omega) \cdot\left[\mathbf{E}_{\mathrm{o}}(\mathbf{q}, \omega)+\mathbf{G}(\mathbf{q} ; \omega) \cdot \mathbf{P}_{1}(\mathbf{q}, \omega)+\mathbf{G}(\mathbf{q} ; \omega) \cdot \mathbf{P}_{2}(\mathbf{q}, \omega)\right],
\end{aligned}
$$

In matrix form, we have

$$
\left(\begin{array}{cc}
\chi_{1}(\mathbf{q} ; \omega)^{-1}-\mathbf{G}(\mathbf{q} ; \omega) & -\mathbf{G}(\mathbf{q} ; \omega) \\
-\mathbf{G}(\mathbf{q} ; \omega) & \chi_{2}(\mathbf{q} ; \omega)^{-1}-\mathbf{G}(\mathbf{q} ; \omega)
\end{array}\right)\left(\begin{array}{l}
\mathbf{P}_{1}(\mathbf{q}, \omega) \\
\mathbf{P}_{2}(\mathbf{q}, \omega)
\end{array}\right)=\left(\begin{array}{l}
\mathbf{E}_{0}(\mathbf{q}, \omega) \\
\mathbf{E}_{0}(\mathbf{q}, \omega)
\end{array}\right)
$$

If we assume the self-interaction has already been taken into account into the definitions of $\chi_{1}$ and $\chi_{2}$, we then have

$$
\left(\begin{array}{cc}
\chi_{1}(\mathbf{q} ; \omega)^{-1} & -\mathbf{G}(\mathbf{q} ; \omega) \\
-\mathbf{G}(\mathbf{q} ; \omega) & \chi_{2}(\mathbf{q} ; \omega)^{-1}
\end{array}\right)\left(\begin{array}{c}
\mathbf{P}_{1}(\mathbf{q}, \omega) \\
\mathbf{P}_{2}(\mathbf{q}, \omega)
\end{array}\right)=\left(\begin{array}{l}
\mathbf{E}_{0}(\mathbf{q}, \omega) \\
\mathbf{E}_{0}(\mathbf{q}, \omega)
\end{array}\right)
$$

This matrix equation is the main result of this section. The coupling factor $\mathbf{G}(\mathbf{q}, \omega)$ may be found either empirically or through rigorous electrodynamic calculations. In the following, we detail the necessary steps to recover Eqn. (2) from the main text. Starting with Eqn. (11), the matrix is inverted to obtain,

$$
\left(\begin{array}{l}
\mathbf{P}_{1}(\mathbf{q}, \omega) \\
\mathbf{P}_{2}(\mathbf{q}, \omega)
\end{array}\right)=\frac{1}{\chi_{1}^{-1}(\mathbf{q}, \omega) \chi_{2}^{-1}(\mathbf{q}, \omega)-\mathbf{G}^{2}(\mathbf{q}, \omega)}\left(\begin{array}{cc}
\chi_{2}^{-1}(\mathbf{q}, \omega) & \mathbf{G}(\mathbf{q} ; \omega) \\
\mathbf{G}(\mathbf{q} ; \omega) & \chi_{1}^{-1}(\mathbf{q}, \omega)
\end{array}\right)\left(\begin{array}{l}
\mathbf{E}_{0}(\mathbf{q}, \omega) \\
\mathbf{E}_{0}(\mathbf{q}, \omega)
\end{array}\right)
$$

The top left element on the right hand side corresponds to the projected polarizability of the $2 \mathrm{D}$ material, written explicitly as:

$$
\chi_{1}^{\prime}(\mathbf{q}, \omega)=\frac{\chi_{2}^{-1}(\mathbf{q}, \omega)}{\chi_{1}^{-1}(\mathbf{q}, \omega) \chi_{2}^{-1}(\mathbf{q}, \omega)-\mathbf{G}^{2}(\mathbf{q}, \omega)}=\frac{1}{\chi_{1}^{-1}(\mathbf{q}, \omega)-\mathbf{G}^{2}(\mathbf{q}, \omega) \chi_{2}(\mathbf{q}, \omega)}
$$


This final expression is in complete agreement with Eqn. (2) of the main text with substitutions $\chi_{1}(\mathbf{q} ; \omega) \rightarrow \chi_{2 \mathrm{D}}, \chi_{2}(\mathbf{q} ; \omega) \rightarrow \chi_{\text {sub }}, \mathbf{G}(\mathbf{q} ; \omega) \rightarrow-\Delta$, and $\mathbf{E}_{\mathrm{o}}(\mathbf{q} ; \omega) \rightarrow \mathbf{E}_{\text {ext }} \rightarrow \varphi_{\text {ext }}$

\subsection{Coupled induced density model}

The above model is an electrodynamic model which takes into account the vectorial nature of the electric fields. In the following, we present an equivalent quasi-static model for coupled induced densities. The following model can be understood on its own, however, it is important to note that it can be derived from the coupled polarizability model in the quasi-static regime where all relevant distances are much smaller than any of the transition wavelengths of the susceptibilities, $\mathrm{d} \ll \lambda$. In this formulation, the induced densities for the $2 \mathrm{D}$ material and substrate $\delta \rho_{1}(\mathbf{r}, \omega)$ and $\delta \rho_{2}(\mathbf{r}, \omega)$, satisfy

$$
\begin{aligned}
& \delta \rho_{1}(\mathbf{r}, \omega)=\int \mathrm{d}^{3} \mathbf{r}^{\prime} \chi_{1}\left(\mathbf{r}_{1}, \mathbf{r}^{\prime} ; \omega\right) \varphi\left(\mathbf{r}^{\prime}, \omega\right), \\
& \delta \rho_{2}(\mathbf{r}, \omega)=\int \mathrm{d}^{3} \mathbf{r}^{\prime} \chi_{2}\left(\mathbf{r}_{2}, \mathbf{r}^{\prime} ; \omega\right) \varphi\left(\mathbf{r}^{\prime}, \omega\right) .
\end{aligned}
$$

The electrostatic potential at point $\mathbf{r}$ is given by:

$$
\varphi(\mathbf{r}, \omega)=\varphi_{\mathrm{o}}(\mathbf{r}, \omega)+\int \mathrm{d}^{3} \mathbf{r}_{1} \mathrm{v}\left(\mathbf{r}, \mathbf{r}_{1} ; \omega\right) \delta \rho_{1}\left(\mathbf{r}_{1}, \omega\right)+\int \mathrm{d}^{3} \mathbf{r}_{2} \mathrm{v}\left(\mathbf{r}, \mathbf{r}_{2} ; \omega\right) \delta \rho_{2}\left(\mathbf{r}_{2}, \omega\right)
$$

These equations are the quasi-static equivalent of equations (14), (15), and (16). The rest of the derivation follows exactly from the above subsection resulting in the primary equations of the main text.

\subsection{Derivation of coupled polarizabilities in the projector formal- ism}

Using a projector-based formalism, ${ }^{19-21}$ we can describe the Coulombic coupling between the two polarizabilities (monolayer and substrate) as a function of their isolated polarizabilities, 
and the nature of the Coulombic coupling in the presence of the substrate.

Definitions ((q, $\omega)$-dependence of polarizabilities not included for the sake of brevity):

Full system: $\chi_{\mathrm{o}}^{-1} \chi-\mathrm{v} \chi=\mathrm{I}$

$\mathrm{P}_{2 \mathrm{D}}=|2 \mathrm{D}\rangle\left\langle 2 \mathrm{D}\left|=\mathrm{P}_{2 \mathrm{D}}^{2} ; \mathrm{P}_{\mathrm{sub}}=\right| \mathrm{sub}\right\rangle\langle\mathrm{sub}|=\mathrm{P}_{\mathrm{sub}}^{2} ; \mathrm{P}_{2 \mathrm{D}}+\mathrm{P}_{\mathrm{sub}}=\mathrm{I} ; \mathrm{P}_{2 \mathrm{D}} \mathrm{P}_{\mathrm{sub}}=(26)$

No transition dipole between the $2: \chi_{\mathrm{o}}=\left(\begin{array}{cc}\chi_{\mathrm{o}, 2 \mathrm{D}}^{\mathrm{i}} & 0 \\ 0 & \chi_{\mathrm{o}, \mathrm{sub}}^{\mathrm{i}}\end{array}\right)$

$\chi_{\mathrm{o}}^{-1}=\left(\begin{array}{cc}\chi_{\mathrm{o}, 2 \mathrm{D}}^{\mathrm{i},-1} & 0 \\ 0 & \chi_{\mathrm{o}, \mathrm{sub}}^{\mathrm{i},-1}\end{array}\right)$

$\mathrm{P}_{2 \mathrm{D}} \chi_{\mathrm{o}} \mathrm{P}_{2 \mathrm{D}}=\chi_{\mathrm{o}, 2 \mathrm{D}}^{\mathrm{i}} ; \mathrm{P}_{\text {sub }} \chi_{\mathrm{o}} \mathrm{P}_{\text {sub }}=\chi_{\mathrm{o}, \mathrm{sub}}^{\mathrm{i}}$

$\mathrm{P}_{2 \mathrm{D}} \chi_{\mathrm{o}}^{-1} \mathrm{P}_{2 \mathrm{D}}=\chi_{\mathrm{o}, 2 \mathrm{D}}^{\mathrm{i},-1} ; \mathrm{P}_{\text {sub }} \chi_{\mathrm{o}}^{-1} \mathrm{P}_{\text {sub }}=\chi_{\mathrm{o}, \mathrm{sub}}^{\mathrm{i},-1}$

$\mathrm{P}_{2 \mathrm{D}} \chi_{\mathrm{o}}^{-1} \mathrm{P}_{2 \mathrm{D}} \chi_{2 \mathrm{D}}^{\mathrm{i}} \mathrm{P}_{2 \mathrm{D}}-\mathrm{P}_{2 \mathrm{D}} \mathrm{P}_{2 \mathrm{D}} \mathrm{P}_{2 \mathrm{D}} \chi_{2 \mathrm{D}}^{\mathrm{i}} \mathrm{P}_{2 \mathrm{D}}=\mathrm{P}_{2 \mathrm{D}}$

$\mathrm{P}_{\text {sub }} \chi_{\mathrm{o}}^{-1} \mathrm{P}_{\text {sub }} \chi_{\text {sub }}^{\mathrm{i}} \mathrm{P}_{\text {sub }}-\mathrm{P}_{\text {sub }} \mathrm{P}_{\text {sub }} \mathrm{P}_{\text {sub }} \chi_{\text {sub }}^{\mathrm{i}} \mathrm{P}_{\text {sub }}=\mathrm{P}_{\text {sub }}$

Please note that

$$
\chi=\mathrm{P}_{2 \mathrm{D}} \chi \mathrm{P}_{2 \mathrm{D}}+\mathrm{P}_{\mathrm{sub}} \chi \mathrm{P}_{2 \mathrm{D}}+\mathrm{P}_{2 \mathrm{D}} \chi \mathrm{P}_{\mathrm{sub}}+\mathrm{P}_{\mathrm{sub}} \chi \mathrm{P}_{\mathrm{sub}}
$$

Derivation:

Projection on $2 \mathrm{D}$ subspace (i.e. multiplying the definition of the polarizability by $\mathrm{P}_{2 \mathrm{D}}$ 
on both sides)

$$
\begin{aligned}
& \mathrm{P}_{2 \mathrm{D} \chi_{\mathrm{o}}}^{-1} \mathrm{I}_{\chi} \mathrm{P}_{2 \mathrm{D}}-\mathrm{P}_{2 \mathrm{D}} \mathrm{I} \chi \mathrm{P}_{2 \mathrm{D}}=\mathrm{P}_{2 \mathrm{D}} \\
& \mathrm{P}_{2 \mathrm{D}} \chi_{\mathrm{o}}^{-1}\left(\mathrm{P}_{2 \mathrm{D}}+\mathrm{P}_{\text {sub }}\right) \chi \mathrm{P}_{2 \mathrm{D}}-\mathrm{P}_{2 \mathrm{DV}}\left(\mathrm{P}_{2 \mathrm{D}}+\mathrm{P}_{\text {sub }}\right) \chi \mathrm{P}_{2 \mathrm{D}}=\mathrm{P}_{2 \mathrm{D}} \\
& \text { since: } \mathrm{P}_{2 \mathrm{D}} \chi_{\mathrm{o}}^{-1} \mathrm{P}_{\mathrm{sub}}=0 \\
& \chi_{\mathrm{o}, 2 \mathrm{D}}^{\mathrm{i},-1} \mathrm{P}_{2 \mathrm{D}} \chi \mathrm{P}_{2 \mathrm{D}}-\mathrm{P}_{2 \mathrm{DV}}\left(\mathrm{P}_{2 \mathrm{D}}+\mathrm{P}_{\text {sub }}\right) \chi \mathrm{P}_{2 \mathrm{D}}=\mathrm{P}_{2 \mathrm{D}} \\
& {\left[\chi_{\mathrm{o}, 2 \mathrm{D}}^{\mathrm{i},-1}-\mathrm{P}_{2 \mathrm{D}} \mathrm{P}_{2 \mathrm{D}}\right] \mathrm{P}_{2 \mathrm{D}} \chi \mathrm{P}_{2 \mathrm{D}}=\mathrm{P}_{2 \mathrm{D}}+\mathrm{P}_{2 \mathrm{D}} \mathrm{P}_{\text {sub }} \mathrm{P}_{\text {sub }} \chi \mathrm{P}_{2 \mathrm{D}}} \\
& \mathrm{P}_{2 \mathrm{D}} \chi \mathrm{P}_{2 \mathrm{D}}=\chi_{2 \mathrm{D}}^{\mathrm{i}}\left[\mathrm{P}_{2 \mathrm{D}}+\mathrm{P}_{2 \mathrm{D}} \mathrm{P}_{\text {sub }} \mathrm{P}_{\text {sub }} \chi \mathrm{P}_{2 \mathrm{D}}\right]
\end{aligned}
$$

We now need to find an expression for the matrix element $\mathrm{P}_{\text {sub }} \chi(\mathbf{q}, \omega) \mathrm{P}_{2 \mathrm{D}}$, which we can find by multiplying the total polarizability on the left by $\mathrm{P}_{\text {sub }}$ and on the right by $\mathrm{P}_{2 \mathrm{D}}$ :

$$
\begin{aligned}
& \mathrm{P}_{\text {sub }} \chi_{\mathrm{o}}^{-1} \mathrm{I}_{\chi} \mathrm{P}_{2 \mathrm{D}}-\mathrm{P}_{\text {sub }} \mathrm{vI} \chi \mathrm{P}_{2 \mathrm{D}}=0 \\
& \mathrm{P}_{\text {sub }} \chi_{\mathrm{o}}^{-1} \mathrm{P}_{\text {sub }} \chi \mathrm{P}_{2 \mathrm{D}}-\mathrm{P}_{\text {sub }} \mathrm{P}_{\text {sub }} \chi \mathrm{P}_{2 \mathrm{D}}=\mathrm{P}_{\text {sub }} \mathrm{v} \mathrm{P}_{2 \mathrm{D}} \chi \mathrm{P}_{2 \mathrm{D}} \\
& {\left[\chi_{\mathrm{o}, \mathrm{sub}}^{\mathrm{i},-1}-\mathrm{P}_{\text {sub }} v \mathrm{P}_{\text {sub }}\right] \mathrm{P}_{\text {sub }} \chi \mathrm{P}_{2 \mathrm{D}}=\mathrm{P}_{\text {sub }} \mathrm{P}_{2 \mathrm{D}} \chi \mathrm{P}_{2 \mathrm{D}}} \\
& \mathrm{P}_{\text {sub }} \chi \mathrm{P}_{2 \mathrm{D}}=\chi_{\text {sub }}^{\mathrm{i}} \mathrm{P}_{\text {sub }}{ } \mathrm{P}_{2 \mathrm{D}} \chi \mathrm{P}_{2 \mathrm{D}}
\end{aligned}
$$

Adding the last expression (34) in the eq. (33) for $\mathrm{P}_{2 \mathrm{D}} \chi \mathrm{P}_{2 \mathrm{D}}$, we get the final expression:

$$
\begin{array}{r}
\mathrm{P}_{2 \mathrm{D}} \chi \mathrm{P}_{2 \mathrm{D}}=\mathrm{P}_{2 \mathrm{D} \chi} \chi_{2 \mathrm{D}}^{\mathrm{i}} \mathrm{P}_{2 \mathrm{D}}+\mathrm{P}_{2 \mathrm{D}} \chi_{2 \mathrm{D}}^{\mathrm{i}} \mathrm{P}_{2 \mathrm{D}} \mathrm{P}_{\text {sub }} \chi_{\text {sub }}^{\mathrm{i}} \mathrm{P}_{\text {sub }} \mathrm{P}_{2 \mathrm{D}} \chi \mathrm{P}_{2 \mathrm{D}} \\
\mathrm{P}_{2 \mathrm{D} \chi} \chi \mathrm{P}_{2 \mathrm{D}}-\mathrm{P}_{2 \mathrm{D} \chi} \chi_{2 \mathrm{D}}^{\mathrm{i}} \mathrm{P}_{2 \mathrm{D}} \mathrm{P}_{\text {sub }} \chi_{\text {sub }}^{\mathrm{i}} \mathrm{P}_{\text {sub }} \mathrm{P}_{2 \mathrm{D}} \chi \mathrm{P}_{2 \mathrm{D}}=\mathrm{P}_{2 \mathrm{D}} \chi_{2 \mathrm{D}}^{\mathrm{i}} \mathrm{P}_{2 \mathrm{D}} \\
\mathrm{P}_{2 \mathrm{D}} \chi_{2 \mathrm{D}}^{\mathrm{i},-1} \mathrm{P}_{2 \mathrm{D}} \chi \mathrm{P}_{2 \mathrm{D}}-\mathrm{P}_{2 \mathrm{D}} \mathrm{P}_{\text {sub }} \chi_{\text {sub }}^{\mathrm{i}} \mathrm{P}_{\text {sub }} \mathrm{P}_{2 \mathrm{D}} \chi \mathrm{P}_{2 \mathrm{D}}=\mathrm{P}_{2 \mathrm{D}} \\
\mathrm{P}_{2 \mathrm{D} \chi} \mathrm{P}_{2 \mathrm{D}}=\mathrm{P}_{2 \mathrm{D}}\left[\mathrm{P}_{2 \mathrm{D}} \chi_{2 \mathrm{D}}^{\mathrm{i},-1} \mathrm{P}_{2 \mathrm{D}}-\mathrm{P}_{2 \mathrm{D}} \mathrm{P}_{\text {sub }} \chi_{\text {sub }}^{\mathrm{i}} \mathrm{P}_{\text {sub }} \mathrm{P}_{2 \mathrm{D}}\right]^{-1} \mathrm{P}_{2 \mathrm{D}}
\end{array}
$$




\subsection{Local expression of plasmon resonances in the quasistatic regime}

There are 4 regions in space, with dielectric functions $\varepsilon_{0}$, the permittivity of the region above borophene, $\varepsilon_{\mathrm{B}}$, permittivity of borophene, $\varepsilon_{\mathrm{i}}$, the permittivity of the insulating layer, and $\varepsilon_{\mathrm{S}}$, the permittivity of the substrate.

$\varphi_{0}, \varphi_{\mathrm{B}}, \varphi_{\mathrm{i}}$, and $\varphi_{\mathrm{S}}$ are the respective solutions for the potential in the different regions of space that must satisfy boundary conditions.

For the boundary conditions, the potential and the permittivity-weighted derivative of the potential must be equal on either side of each interface. Thus, for the BCs, we have:

$$
\begin{aligned}
\left.\varphi_{0}\right|_{\mathrm{z}=\mathrm{z}_{2}} & =\left.\varphi_{\mathrm{B}}\right|_{\mathrm{z}=\mathrm{z}_{2}} \\
\left.\varphi_{\mathrm{B}}\right|_{\mathrm{z}=\mathrm{z}_{1}} & =\left.\varphi_{\mathrm{i}}\right|_{\mathrm{z}=\mathrm{z}_{1}} \\
\left.\varphi_{\mathrm{i}}\right|_{\mathrm{z}=0} & =\left.\varphi_{\mathrm{s}}\right|_{\mathrm{z}=0} \\
\left.\varepsilon_{0} \frac{\partial \varphi_{0}}{\partial \mathrm{z}}\right|_{\mathrm{z}=\mathrm{z}_{2}} & =\left.\varepsilon_{\mathrm{B}} \frac{\partial \varphi_{\mathrm{B}}}{\partial \mathrm{z}}\right|_{\mathrm{z}=\mathrm{z}_{2}} \\
\left.\varepsilon_{\mathrm{B}} \frac{\partial \varphi_{\mathrm{B}}}{\partial \mathrm{z}}\right|_{\mathrm{z}=\mathrm{z}_{1}} & =\left.\varepsilon_{\mathrm{i}} \frac{\partial \varphi_{\mathrm{i}}}{\partial \mathrm{z}}\right|_{\mathrm{z}=\mathrm{z}_{1}} \\
\left.\varepsilon_{\mathrm{i}} \frac{\partial \varphi_{\mathrm{i}}}{\partial \mathrm{z}}\right|_{\mathrm{z}=0} & =\left.\varepsilon_{\mathrm{s}} \frac{\partial \varphi_{\mathrm{S}}}{\partial \mathrm{z}}\right|_{\mathrm{z}=0}
\end{aligned}
$$

The product solutions for the Laplace equation are $(\mathbf{k} \cdot \mathbf{r}$ refers to $\mathbf{k}$ and $\mathbf{r}$ in the $\mathrm{x}-\mathrm{y}$ plane):

$$
\begin{aligned}
\varphi_{0} & =\mathrm{Ae}^{\mathrm{j} \mathbf{k} \cdot \mathbf{r}-\mathrm{kz}} \\
\varphi_{\mathrm{B}} & =\left(\mathrm{Be}^{-\mathrm{kz}}+\mathrm{Ce}^{+\mathrm{kz}}\right) \mathrm{e}^{\mathrm{j} \mathbf{k} \cdot \mathbf{r}} \\
\varphi_{\mathrm{i}} & =\left(\mathrm{De} \mathrm{e}^{-\mathrm{kz}}+\mathrm{Ee}^{+\mathrm{kz}}\right) \mathrm{e}^{\mathrm{j} \mathbf{k} \cdot \mathbf{r}} \\
\varphi_{\mathrm{S}} & =\mathrm{Fe}^{\mathrm{j} \mathbf{k} \cdot \mathbf{r}+\mathrm{kz}}
\end{aligned}
$$


The boundary conditions are satisfied if the following relations are valid:

$$
\begin{aligned}
& \mathrm{Ae}^{-\mathrm{kz} z_{2}}=\mathrm{Be}^{-\mathrm{kz} z_{2}}+\mathrm{Ce}^{+\mathrm{kz} z_{2}} \\
& \mathrm{Be}^{-\mathrm{kz} z_{1}}+\mathrm{Ce}^{+\mathrm{kz} z_{1}}=\mathrm{De}^{-\mathrm{kz} z_{1}}+\mathrm{Ee}^{+\mathrm{kz} z_{1}} \\
& \mathrm{D}+\mathrm{E}=\mathrm{F} \\
& \varepsilon_{0} \mathrm{Ae}^{-\mathrm{kz} z_{2}}=\varepsilon_{\mathrm{B}}\left(\mathrm{Be}^{-\mathrm{kz} z_{2}}-\mathrm{Ce}^{+\mathrm{kz} z_{2}}\right) \\
& \varepsilon_{\mathrm{B}}\left(\mathrm{Be}^{-\mathrm{kz} z_{1}}-\mathrm{Ce}^{+\mathrm{kz} z_{1}}\right)=\varepsilon_{\mathrm{i}}\left(\mathrm{De}^{-\mathrm{kz} z_{1}}-\mathrm{Ee}^{+\mathrm{kz} z_{1}}\right) \\
& \varepsilon_{\mathrm{i}}(-\mathrm{D}+\mathrm{E})=\varepsilon_{\mathrm{S}} \mathrm{F}
\end{aligned}
$$

Substituting (46) into (49) and (48) into (51), we get:

$$
\begin{aligned}
& \mathrm{Be}^{-\mathrm{kz} z_{1}}+\mathrm{Ce}^{+\mathrm{kz} z_{1}}=\mathrm{De}^{-\mathrm{kz} z_{1}}+\mathrm{Ee}^{+\mathrm{kz} z_{1}} \\
& \varepsilon_{0}\left(\mathrm{Be}^{-\mathrm{kz} z_{2}}+\mathrm{Ce}^{+\mathrm{kz} z_{2}}\right)=\varepsilon_{\mathrm{B}}\left(\mathrm{Be}^{-\mathrm{kz} z_{2}}-\mathrm{Ce}^{+\mathrm{kz} z_{2}}\right) \\
& \varepsilon_{\mathrm{B}}\left(\mathrm{Be}^{-\mathrm{kz}} \mathrm{z}_{1}-\mathrm{Ce}^{+\mathrm{kz_{1 }}}\right)=\varepsilon_{\mathrm{i}}\left(\mathrm{De}^{-\mathrm{kz}_{1}}-\mathrm{Ee}^{+\mathrm{kz}_{1}}\right) \\
& \varepsilon_{\mathrm{i}}(-\mathrm{D}+\mathrm{F})=\varepsilon_{\mathrm{s}}(\mathrm{D}+\mathrm{E})
\end{aligned}
$$

In matrix form, the above set of conditions becomes:

$$
\left[\begin{array}{cccc}
\mathrm{e}_{2}^{-}\left(\varepsilon_{0}-\varepsilon_{\mathrm{B}}\right) & \mathrm{e}_{2}^{+}\left(\varepsilon_{0}+\varepsilon_{\mathrm{B}}\right) & 0 & 0 \\
\mathrm{e}_{1}^{-} & \mathrm{e}_{1}^{+} & -\mathrm{e}_{1}^{-} & -\mathrm{e}_{1}^{+} \\
\varepsilon_{\mathrm{B}} \mathrm{e}_{1}^{-} & -\varepsilon_{\mathrm{B}} \mathrm{e}_{1}^{+} & -\varepsilon_{\mathrm{i}} \mathrm{e}_{1}^{-} & \varepsilon_{\mathrm{i}} \mathrm{e}_{1}^{+} \\
0 & 0 & -\varepsilon_{\mathrm{i}}-\varepsilon_{\mathrm{S}} & \varepsilon_{\mathrm{i}}-\varepsilon_{\mathrm{S}}
\end{array}\right]\left[\begin{array}{l}
\mathrm{B} \\
\mathrm{C} \\
\mathrm{D} \\
\mathrm{E}
\end{array}\right]=\left[\begin{array}{l}
0 \\
0 \\
0 \\
0
\end{array}\right]
$$

where $\mathrm{e}_{\mathrm{n}}^{ \pm} \equiv \mathrm{e}^{ \pm \mathrm{k} \mathrm{z}_{\mathrm{n}}}$ and $\varepsilon_{\mathrm{a}}-\varepsilon_{\mathrm{b}} \equiv \overline{\varepsilon_{\mathrm{ab}}}$ for brevity. This has nontrivial solutions when the determinant of the matrix is 0 . 
The determinant of the above matrix becomes:

$$
\overline{\varepsilon_{0 \mathrm{~B}}} \mathrm{e}_{2}^{-}\left(\bar{\varepsilon}_{\mathrm{si}} \varepsilon_{\mathrm{Bi}}+\mathrm{e}_{2}^{+} \varepsilon_{\mathrm{si}} \overline{\bar{i}_{\mathrm{iB}}}\right)-\varepsilon_{0 \mathrm{~B}} \mathrm{e}_{2}^{+}\left(\mathrm{e}_{1}^{-2} \overline{\varepsilon_{\mathrm{si}} \varepsilon_{\mathrm{iB}}}+\varepsilon_{\mathrm{si}} \varepsilon_{\mathrm{iB}}\right)=0
$$

The roots of this equation give plasmon resonances of the substrate-insulator-boropheneair configuration.

\section{Derivation of the supported polarizabilities}

A derivation of coupled interacting polarizabilities in the quasistatic, local-response, classicalelectrodynamical limit can be equivalently derived as follows. The electron density response function for the $2 \mathrm{D}$ system is given by:

$$
\chi(\mathbf{q}, \omega)=\frac{1}{\chi_{\mathrm{o}}^{-1}(\omega)-\mathrm{v}(\mathbf{q})}
$$

where $\chi_{0}(\omega)$ is the bare response function and $\mathrm{v}(\mathbf{q})$ is the Fourier transform of the Coulomb potential. The Coulomb potential is generally an environment-dependent quantity obeying the decomposition,

$$
\mathrm{v}(\mathbf{q})=\mathrm{v}_{\mathrm{o}}(\mathbf{q})+\mathrm{v}_{\mathrm{scat}}(\mathbf{q}),
$$

where $\mathrm{v}_{\mathrm{o}}(\mathbf{q})$ is the bare (free-space) Coulomb interaction and $\mathrm{v}_{\text {scat }}(\mathbf{q})$ is the environmentdependent potential. Explicitly, $\mathbf{v}(\mathbf{q})$ is found by solving the Poisson equation for different piecewise regions denoted by their permittivities. This problem has a well-known solution when both $\mathbf{r}$ and $\mathbf{r}^{\prime}$ are on the same side of a single interface. The solution may be written in terms of image charges:

$$
\mathrm{v}\left(\mathbf{r}, \mathbf{r}^{\prime}\right)=\frac{1}{\varepsilon_{\mathrm{b}}} \frac{\mathrm{e}^{2}}{4 \pi\left|\mathbf{r}-\mathbf{r}^{\prime}\right|}+\frac{1}{\varepsilon_{\mathrm{b}}} \frac{\varepsilon_{\mathrm{b}}-\varepsilon_{\text {substrate }}+\varepsilon_{\text {substrate }}}{\varepsilon_{\mathrm{b}}} \frac{\mathrm{e}^{2}}{4 \pi\left|\mathbf{r}-\mathbf{r}^{\prime *}\right|}
$$


where $\mathbf{r}^{*}=(\mathrm{x}, \mathrm{y},-\mathrm{d})$ is the image charge position. This result intuitively shows how charges in Borophene experience a fundamentally different potential due to the existence of image charges in the substrate. Taking the Fourier transform of this quantity, we find

$$
\mathrm{v}(\mathbf{q})=\frac{1}{\varepsilon_{\mathrm{b}}} \frac{\mathrm{e}^{2}}{\mathrm{q}^{2}}+\frac{1}{\varepsilon_{\mathrm{b}}} \frac{\varepsilon_{\mathrm{b}}-\varepsilon_{\text {drude }}}{\varepsilon_{\mathrm{b}}+\varepsilon_{\text {drude }}} \frac{\mathrm{e}^{2}}{\mathrm{q}^{2}} \mathrm{e}^{-2 \mathrm{qd}} .
$$

These equations can be used to provide a qualitative understanding of the full DFT simulations, specifically illustrating how the electron density response function is modified by the environment.

\section{Independent-particle polarizabilities}

Figures 1-5 present the independent-particle (IP) polarizabilities of $\mathrm{Ag}(111)$ and borophene adsorbed on $\operatorname{Ag}(111)$ with varying distances.
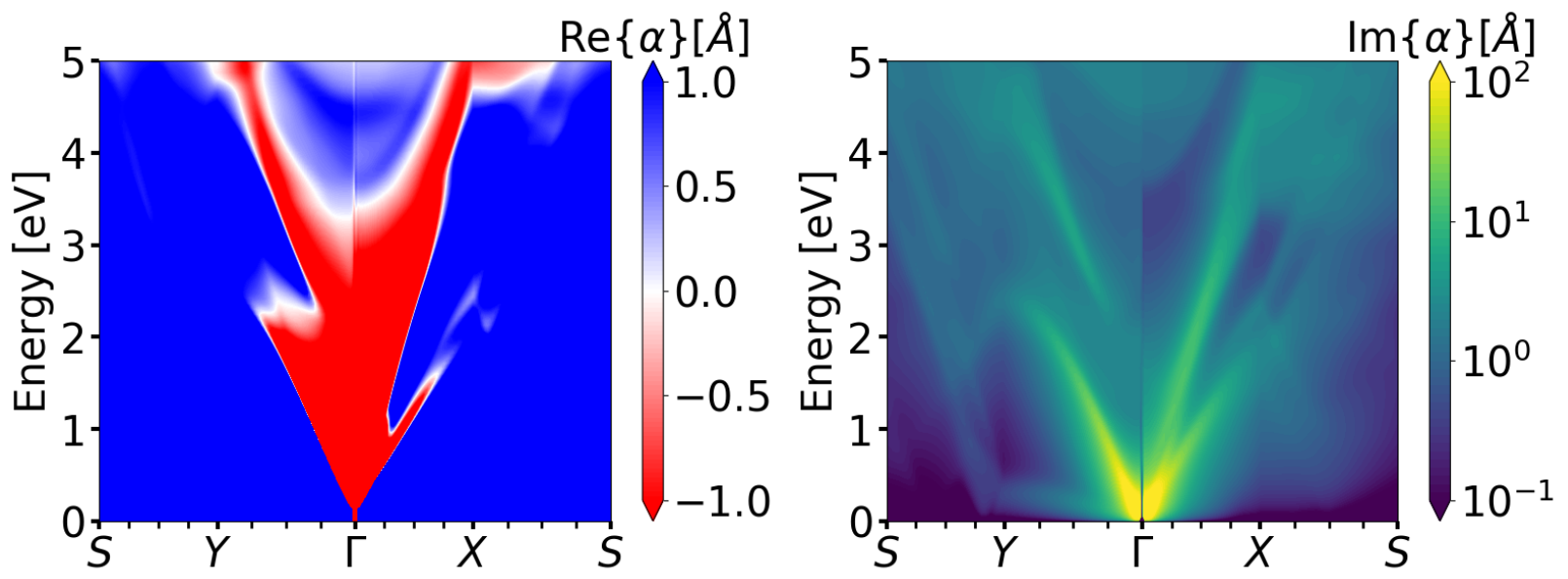

Figure 2: Independent-particle polarizability of freestanding borophene 


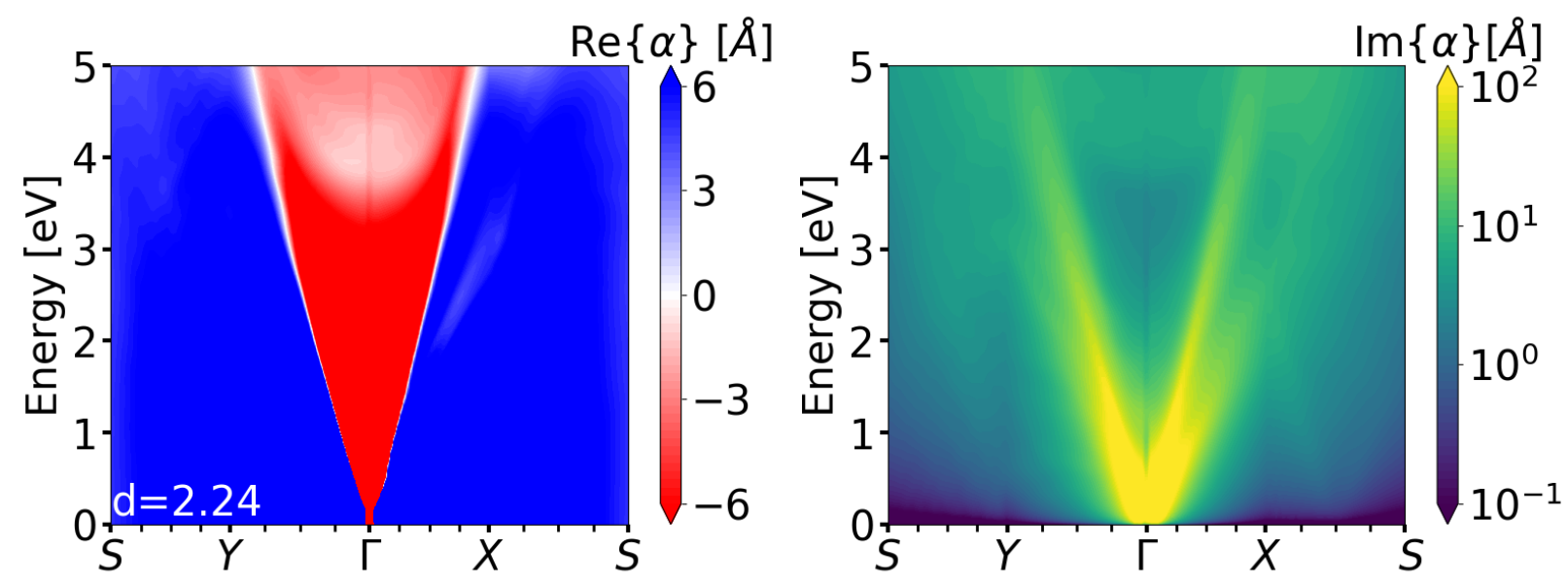

Figure 3: Independent-particle polarizability of borophene on $\mathrm{Ag}(111)$ at equilibrium (2.24 $\AA$ above the surface).

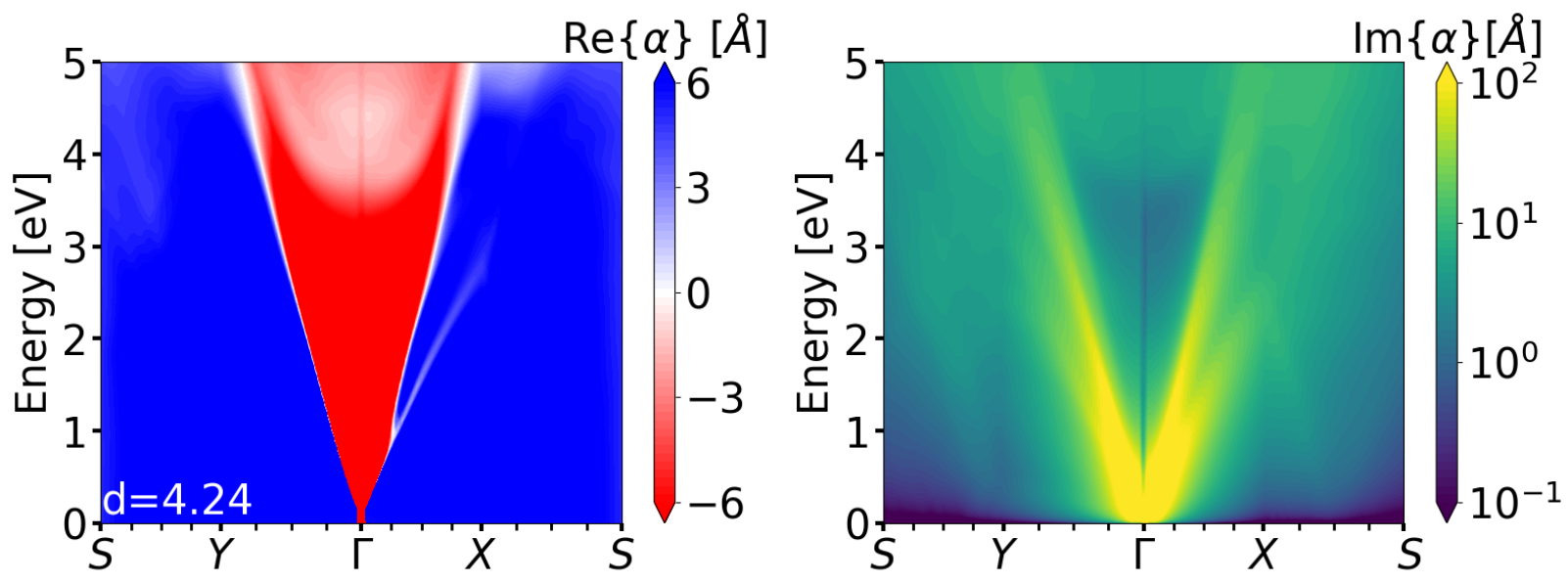

Figure 4: Independent-particle polarizability of borophene on $\mathrm{Ag}(111)$ with separation 4.24 A. 


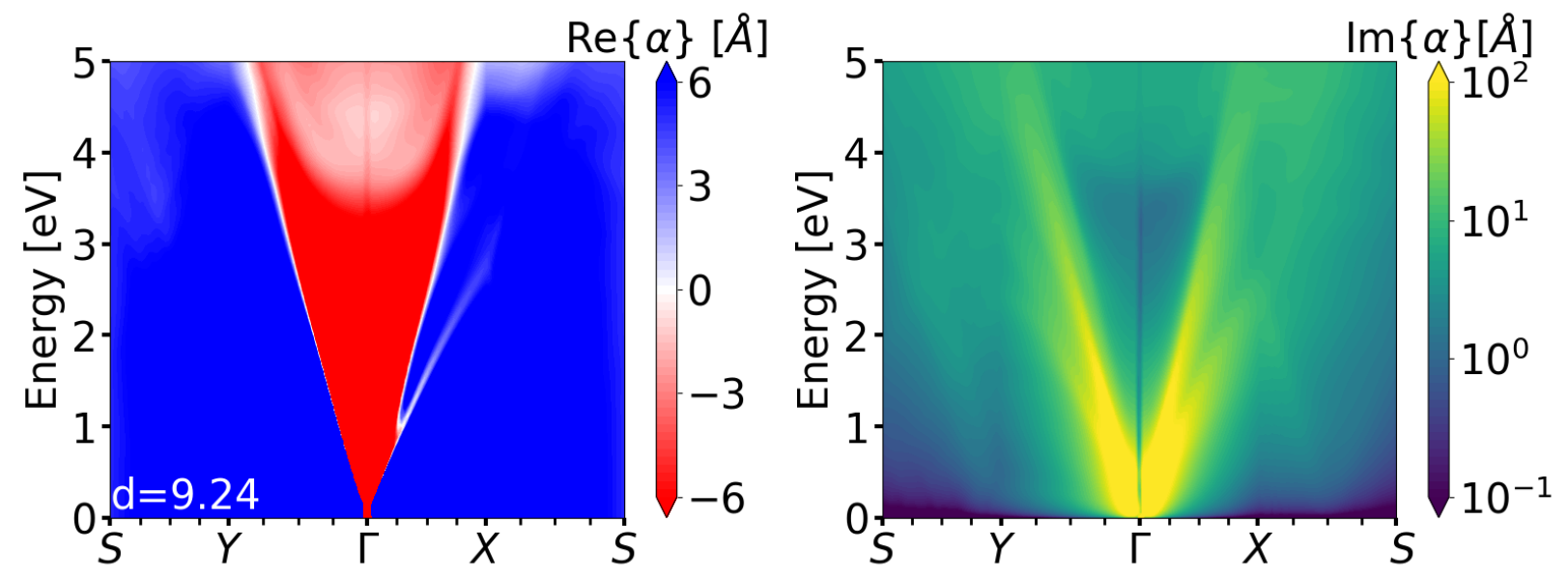

Figure 5: Independent-particle polarizability of borophene on $\mathrm{Ag}(111)$ with separation 9.24 A.

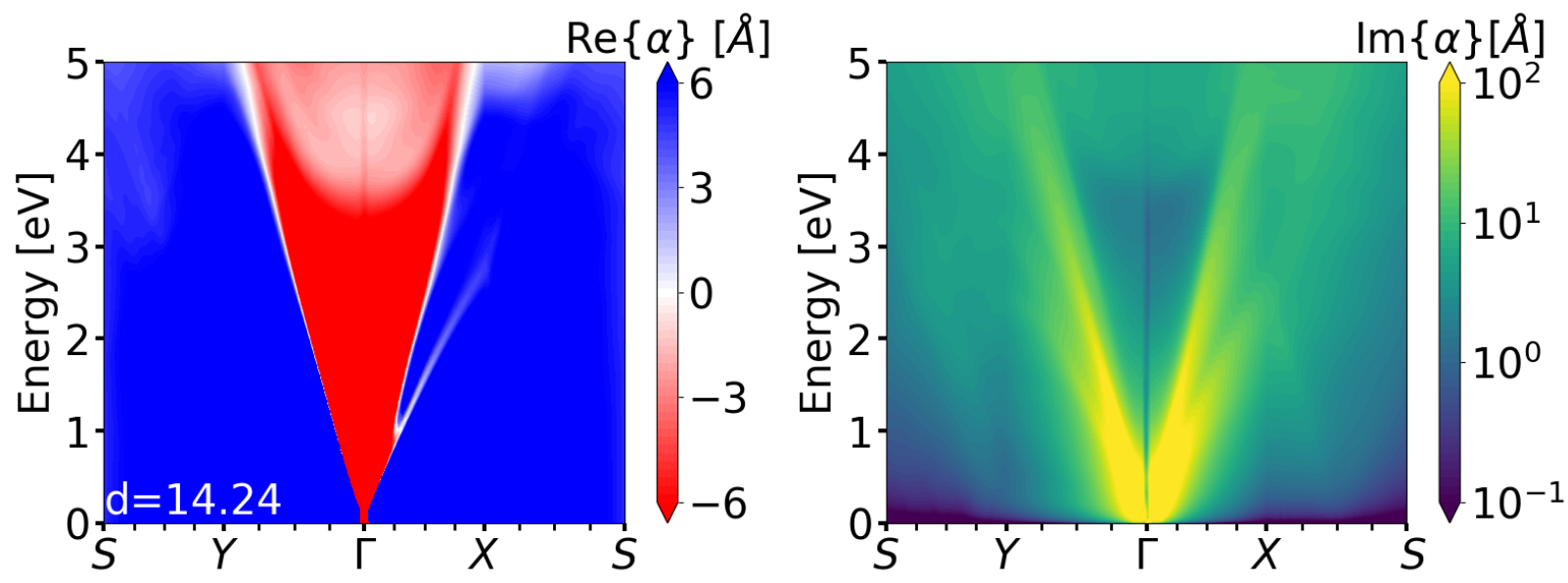

Figure 6: Independent-particle polarizability of borophene on $\mathrm{Ag}(111)$ with separation 14.24 $\AA$.

\section{Conventional bandpath}

The Brillouin Zone (BZ) of our calculation supercell is shown below. 


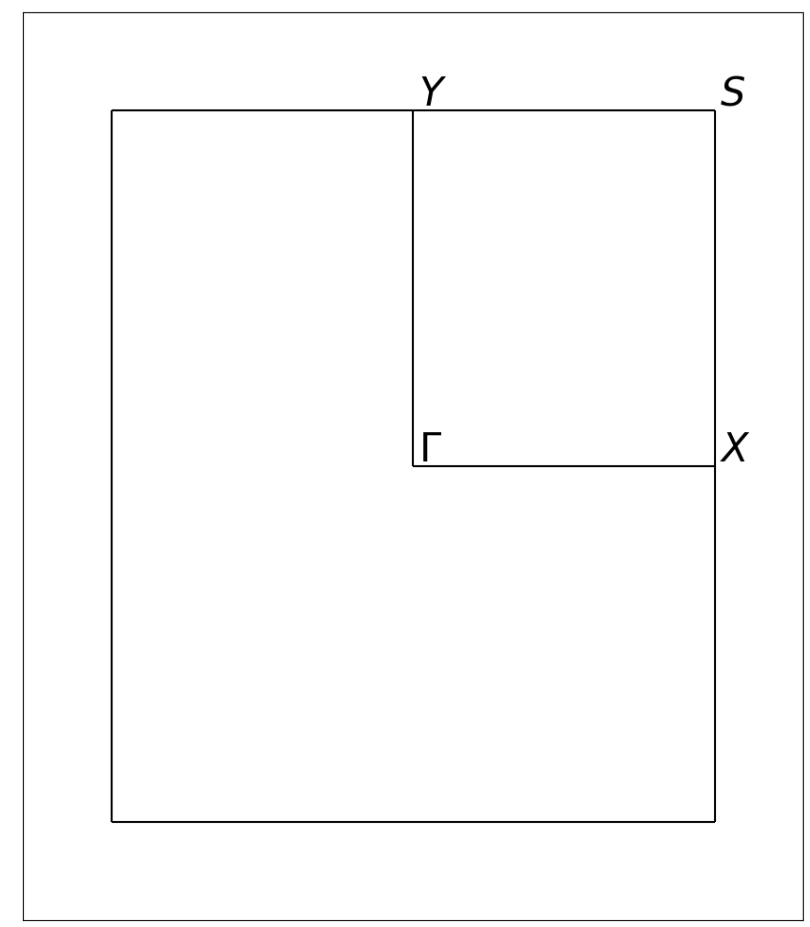

Figure 7: The bandpath of the BZ of the borophene $2 \times 1$ supercell, with high-symmetry points labelled.

\section{Bandpaths for polarizability 'wedges'}

For bandstructure calculations, a special, non-conventional path in the BZ, shown in Figure 7, was chosen to assign approximate representative transitions (in the independent-particle approximation) that are responsible for the non-monotonocity of the single-particle continuum of borophene. The 'wedges' in the single-particle continuum onsets refer to the regions in energy-momentum space, $\sim 1 \mathrm{eV}$ along $\mathrm{G}-\mathrm{X}$ and $\sim 2.5 \mathrm{eV}$ along $\mathrm{G}-\mathrm{Y}$, see SI figure 1, where the poles in the independent-particle polarizability are non-monotonic due to the electronic structure of borophene. 


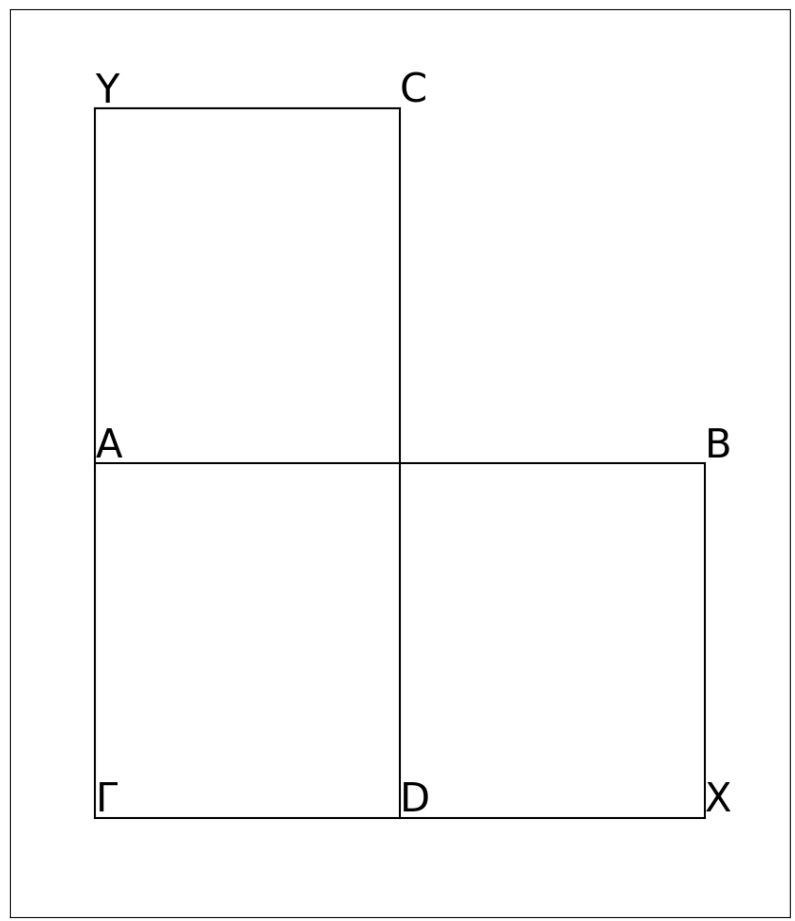

Figure 8: The bandpath in the BZ mapped out to find the source of the independent-particle transition 'wedges' of the polarizabilities.

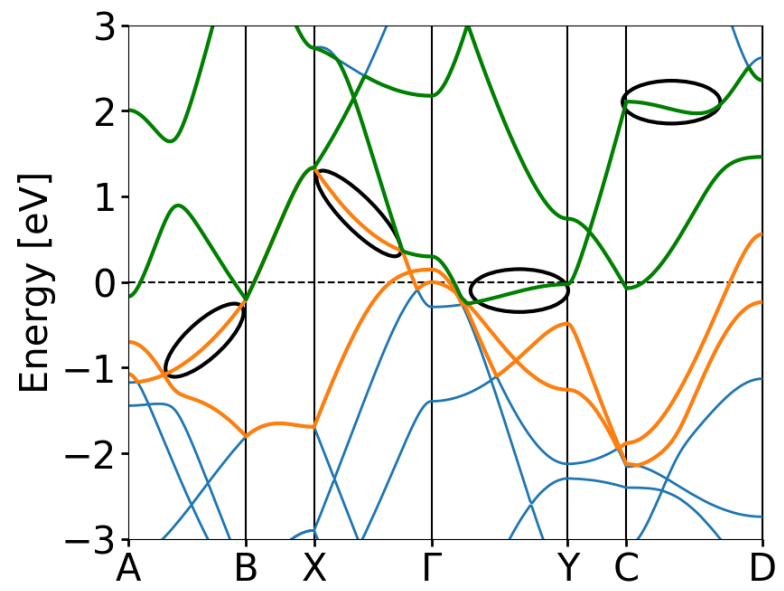

Figure 9: The borophene calculated bandstructure along the path shown in Figure 7.

Figure 8 shows a representative set of transitions that could contribute to the independent particle transitions shown in Figure 1. The black circles show possible transitions along $\Gamma-\mathrm{X}$ and $\Gamma$-Y where the momentum dependence and energy range are consistent with the poles in the IP spectrum. In particular, transitions from $\mathrm{AB}$ to $\Gamma-\mathrm{X}$ range approximately from 
0.5-2 eV while the transitions from $\Gamma-Y$ to $\mathrm{C}-\mathrm{D}$ range approximately $2-2.5 \mathrm{eV}$. For example, there is a clear $\sim 2 \mathrm{eV}$ shift between Gamma-Y and CD flat band, indicating an interband transition at that energy for $\mathrm{a} \sim 0.5 \Gamma-\mathrm{X}$ momentum.

\section{Charge transfer at the borophene- $\operatorname{Ag}(111)$ interface}

\subsection{Psuedo-charge density at borophene/Ag(111)}

Figure 9 presents the pseudo-charge density at the borophene-Ag(111) interface, showing weak charge transfer between the borophene and the $\operatorname{Ag}(111)$ slab, at equilibrium.

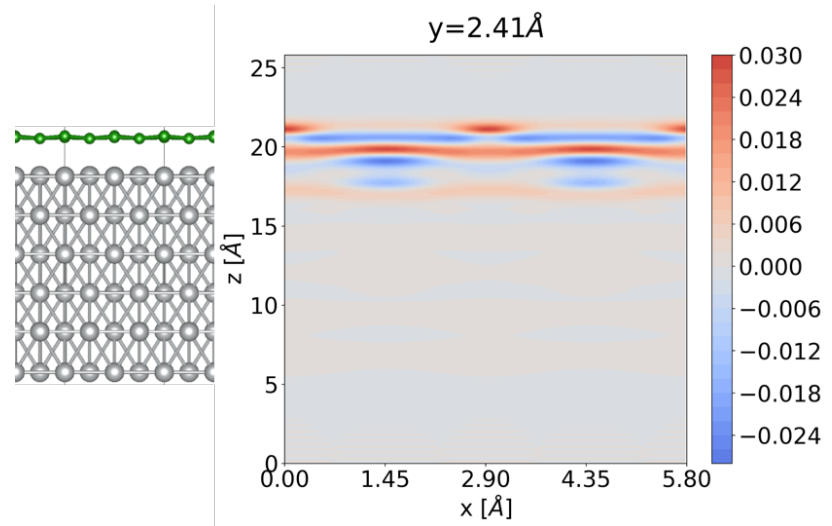

Figure 10: Pseudo-charge density contour plot showing charge transfer between borophene and $\operatorname{Ag}(111)$ at equilibrium. The units of charge transfer are in electronic charge. The geometry on the left is used to guide the eye on the approximate location of the atoms around which charge transfer occurs.

\subsection{Bader analysis of charge transfer between borophene and sub- strates}

We calculate the average charge transfer from the metal substrate to borophene for borophene/Ag(111) and borophene/Al(111) using a Bader analysis as shown below. Here, Atoms 1-24 are the metal and 25-34 are boron. The average charge transfer from metal to borophene is 0.02 electrons/boron on $\mathrm{Ag}(111)$ and 0.2 electrons/boron on $\mathrm{Al}(111)$. 
Borophene/ $\operatorname{Ag}(111)$ :

\begin{tabular}{|c|c|c|c|c|c|c|}
\hline \# & $\mathrm{X}$ & $\mathrm{Y}$ & $\mathrm{Z}$ & CHARGE & MIN DIST & ATOMIC VOL \\
\hline 1 & 2.732607 & 1.577671 & 13.228083 & 47.021859 & 2.586322 & 349.210264 \\
\hline 2 & 8.197821 & 1.577671 & 13.228083 & 47.021864 & 2.586314 & 349.210264 \\
\hline 3 & 0.000000 & 6.310686 & 13.228083 & 46.997656 & 2.586318 & 358.795637 \\
\hline 4 & 5.465214 & 6.310686 & 13.228083 & 47.051296 & 2.586311 & 365.609816 \\
\hline 5 & 0.000000 & 3.155343 & 17.690411 & 46.960196 & 2.497729 & 111.433185 \\
\hline 6 & 5.465214 & 3.155343 & 17.690411 & 47.005321 & 2.497729 & 113.507473 \\
\hline 7 & 2.732607 & 7.888357 & 17.690411 & 46.981808 & 2.497742 & 112.399935 \\
\hline 8 & 8.197821 & 7.888357 & 17.690411 & 46.981813 & 2.497742 & 112.399935 \\
\hline 9 & 0.000000 & 0.043286 & 21.948239 & 46.972272 & 2.496781 & 109.235706 \\
\hline 10 & 5.465214 & 0.043286 & 21.948239 & 47.018818 & 2.496773 & 111.331113 \\
\hline 11 & 2.732607 & 4.781114 & 21.942677 & 46.995131 & 2.493499 & 110.243519 \\
\hline 12 & 8.197821 & 4.781114 & 21.942677 & 46.995135 & 2.493492 & 110.243519 \\
\hline 13 & 2.732607 & 1.679920 & 26.216016 & 47.001607 & 2.488028 & 110.839525 \\
\hline 14 & 8.197821 & 1.679920 & 26.216016 & 47.001612 & 2.488022 & 110.839525 \\
\hline 15 & 0.000000 & 6.402162 & 26.225164 & 46.978152 & 2.491755 & 109.612316 \\
\hline 16 & 5.465214 & 6.402162 & 26.225164 & 47.023711 & 2.491749 & 111.649061 \\
\hline 17 & 0.000000 & 3.301055 & 30.522950 & 46.975924 & 2.497482 & 109.696789 \\
\hline 18 & 5.465214 & 3.301055 & 30.522950 & 47.022294 & 2.497482 & 111.813315 \\
\hline 19 & 2.732607 & 8.033715 & 30.491170 & 47.005131 & 2.476480 & 110.222401 \\
\hline 20 & 8.197821 & 8.033715 & 30.491170 & 47.005158 & 2.476474 & 110.223574 \\
\hline 21 & 0.000000 & 0.151798 & 34.739306 & 46.928654 & 2.416415 & 105.410943 \\
\hline 22 & 5.465214 & 0.151798 & 34.739306 & 46.973964 & 2.416413 & 107.418357 \\
\hline 23 & 2.732607 & 4.969319 & 34.807936 & 46.935856 & 2.362726 & 107.440648 \\
\hline 24 & 8.197821 & 4.969319 & 34.807936 & 46.935861 & 2.362725 & 107.440648 \\
\hline 25 & 0.000000 & 7.839397 & 39.090490 & 4.868166 & 1.287349 & 172.916839 \\
\hline 26 & 0.000000 & 1.553962 & 39.196954 & 4.495171 & 1.340883 & 169.234038 \\
\hline 27 & 0.000000 & 4.769562 & 38.990191 & 4.772053 & 1.284290 & 149.023802 \\
\hline 28 & 2.732607 & 9.398242 & 38.913688 & 5.451452 & 1.536443 & 110.845391 \\
\hline 29 & 2.732607 & 3.195139 & 38.885187 & 5.515220 & 1.518533 & 106.714412 \\
\hline 30 & 5.465214 & 7.839397 & 39.090490 & 4.867573 & 1.287337 & 173.724029 \\
\hline 31 & 5.465214 & 1.553962 & 39.196954 & 4.495109 & 1.340874 & 169.454607 \\
\hline 32 & 5.465214 & 4.769562 & 38.990191 & 4.772000 & 1.284279 & 150.171231 \\
\hline 33 & 8.197821 & 9.398242 & 38.913688 & 5.451452 & 1.536432 & 110.845391 \\
\hline 34 & 8.197821 & 3.195139 & 38.885187 & 5.515220 & 1.518521 & 106.714412 \\
\hline & & & & & & \\
\hline \multicolumn{3}{|c|}{ VACUUM CHARGE: } & 0.0000 & & & \\
\hline \multirow{2}{*}{\multicolumn{2}{|c|}{ VACUUM VOLUME: }} & & 0.0000 & & & \\
\hline \multicolumn{2}{|c|}{ NUMBER OF ELEC? } & & 177.9945 & & & \\
\hline
\end{tabular}

Borophene/Al(111):

\begin{tabular}{|c|c|c|c|c|c|c|}
\hline \# & $\mathrm{X}$ & $\mathrm{Y}$ & Z & CHARGE & MIN DIST & ATOMIC VOL \\
\hline 1 & 4830743 & 1792582 & 19705365 & 12989898 & 2466130 & $509 \quad 171427$ \\
\hline 2 & 10.242508 & 1.792582 & 19.705365 & 12.989898 & 2.466121 & 509.177094 \\
\hline 3 & 2.124861 & 6.479307 & 19.705365 & 12.983772 & 2.475945 & 502.439828 \\
\hline
\end{tabular}




\begin{tabular}{|c|c|c|c|c|c|c|}
\hline 4 & 7.536626 & 6.479307 & 19.705365 & 12.983771 & 2.475953 & 502.438694 \\
\hline 5 & 2.124861 & 3.354823 & 24.124052 & 13.001060 & 2.446072 & 110.556235 \\
\hline 6 & 7.536626 & 3.354823 & 24.124052 & 13.001061 & 2.446064 & 110.556235 \\
\hline 7 & 4.830743 & 8.041549 & 24.124052 & 12.992824 & 2.403581 & 110.136856 \\
\hline 8 & 10.242508 & 8.041549 & 24.124052 & 12.992824 & 2.403572 & 110.136856 \\
\hline 9 & 2.065664 & 0.153701 & 28.387819 & 12.996851 & 2.404526 & 108.353928 \\
\hline 10 & 7.477428 & 0.153701 & 28.387819 & 12.996851 & 2.404519 & 108.353928 \\
\hline 11 & 4.773533 & 4.839896 & 28.420384 & 12.991552 & 2.427041 & 108.616890 \\
\hline 12 & 10.185298 & 4.839896 & 28.420384 & 12.991656 & 2.427032 & 108.620291 \\
\hline 13 & 4.727668 & 1.568654 & 32.666682 & 13.024299 & 2.453295 & 109.338902 \\
\hline 14 & 10.139432 & 1.568654 & 32.666682 & 13.024194 & 2.453288 & 109.335502 \\
\hline 15 & 2.026037 & 6.283446 & 32.718797 & 13.045774 & 2.463523 & 110.603840 \\
\hline 16 & 7.437802 & 6.283446 & 32.718797 & 13.045742 & 2.463532 & 110.602707 \\
\hline 17 & 1.992031 & 2.988278 & 36.935219 & 12.915299 & 2.401890 & 106.330708 \\
\hline 18 & 7.403796 & 2.988278 & 36.935219 & 12.915270 & 2.401898 & 106.329575 \\
\hline 19 & 4.718922 & 7.720241 & 37.032929 & 12.921561 & 2.416483 & 107.943617 \\
\hline 20 & 10.130687 & 7.720241 & 37.032929 & 12.921526 & 2.416490 & 107.942484 \\
\hline 21 & 1.979350 & 9.094486 & 41.486438 & 12.478655 & 1.629625 & 95.644478 \\
\hline 22 & 7.391115 & 9.094486 & 41.486438 & 12.478717 & 1.629625 & 95.646745 \\
\hline 23 & 4.662599 & 4.319468 & 41.211812 & 12.704594 & 1.657044 & 101.867156 \\
\hline 24 & 10.074363 & 4.319468 & 41.211812 & 12.704599 & 1.657048 & 101.867156 \\
\hline 25 & 1.715441 & 2.828749 & 45.778139 & 4.828456 & 1.287733 & 176.863453 \\
\hline 26 & 1.718041 & 5.988585 & 46.117915 & 4.480929 & 1.339725 & 446.780302 \\
\hline 27 & 1.723458 & 9.143959 & 45.808276 & 5.226291 & 1.239787 & 249.011373 \\
\hline 28 & 4.425459 & 4.390415 & 45.564733 & 5.866019 & 1.484496 & 98.603934 \\
\hline 29 & 4.422454 & 7.610059 & 45.516745 & 5.551970 & 1.525554 & 81.527275 \\
\hline 30 & 7.127206 & 2.828749 & 45.778139 & 4.828843 & 1.287747 & 176.863453 \\
\hline 31 & 7.129805 & 5.988585 & 46.117915 & 4.482194 & 1.339714 & 446.799571 \\
\hline 32 & 7.135223 & 9.143959 & 45.808276 & 5.226308 & 1.239776 & 249.022708 \\
\hline 33 & 9.837224 & 4.390415 & 45.564733 & 5.866325 & 1.484508 & 98.609601 \\
\hline 34 & 9.834219 & 7.610059 & 45.516745 & 5.549277 & 1.525567 & 81.469469 \\
\hline \multicolumn{3}{|c|}{ VACUUM CHARGE: } & .0000 & & & \\
\hline \multicolumn{3}{|c|}{ VACUUM VOLUME: } & 0.0000 & & & \\
\hline \multicolumn{3}{|c|}{ NUMBER } & 1.9989 & & & \\
\hline
\end{tabular}




\section{Influence of a van der Waals correction to geometry on the predicted borophene response}

we show the response function computed optimizing the geometry using the PBE functional ${ }^{3}$ with a DFT-D3 (Becke-Johnson damping) ${ }^{4}$ van der Waals correction. We find the role of van der Waals interactions to be negligible on the response function of borophene on $\operatorname{Ag}(111)$ or $\mathrm{Al}(111)$ because the change in inter-layer distance and borophene corrugation is quite small when compared to our original LDA-optimized geometry. The adsorption distance for borophene is $2.24(2.31) \AA$ on $\mathrm{Ag}(111)$ and 2.33 (2.45) $\AA$ on $\mathrm{Al}(111)$ with LDA (PBED3). The average out-of-plane distance of boron atoms with and without van der Waals corrections is $\sim 0.07 \AA$ and $\sim 0.06 \AA$ respectively, for borophene adsorbed on $\operatorname{Ag}(111)$ and $\sim 0.10 \AA$ and $\sim 0.11 \AA$ respectively, for borophene/ $\mathrm{Al}(111)$.
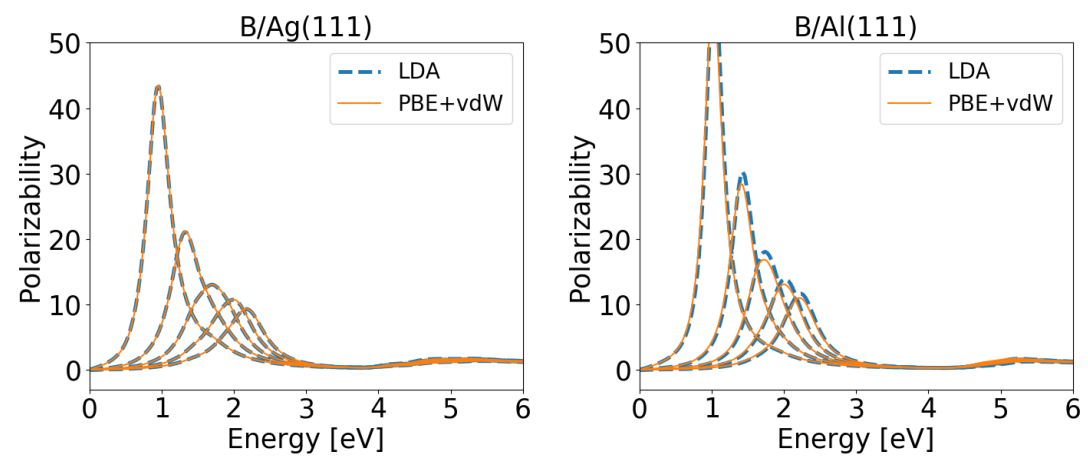

Figure 11: The effect of the van der Waals corrected geometry on the calculated response of isolated borophene for a series of q-vectors and for geometry optimized on $\operatorname{Ag}(111)$ and $\mathrm{Al}(111)$.

\section{Borophene/Ag(111) model fits}

\subsection{Borophene/Ag(111)}

This section presents all of the model fits, along with the data they were fit to, of the 2 $\times 2$ coupled-polarizability model. The transferred momentum and the borophene- $\operatorname{Ag}(111)$ 
distance are in the title of each figure, while the numerical value of $t(\mathbf{q})$ factor is in the plot itself. The dashed lines represent RPA-calculated polarizabilities, and the solid lines represent the model polarizability. The blue and orange lines are the imaginary and real parts of the polarizability, respectively.
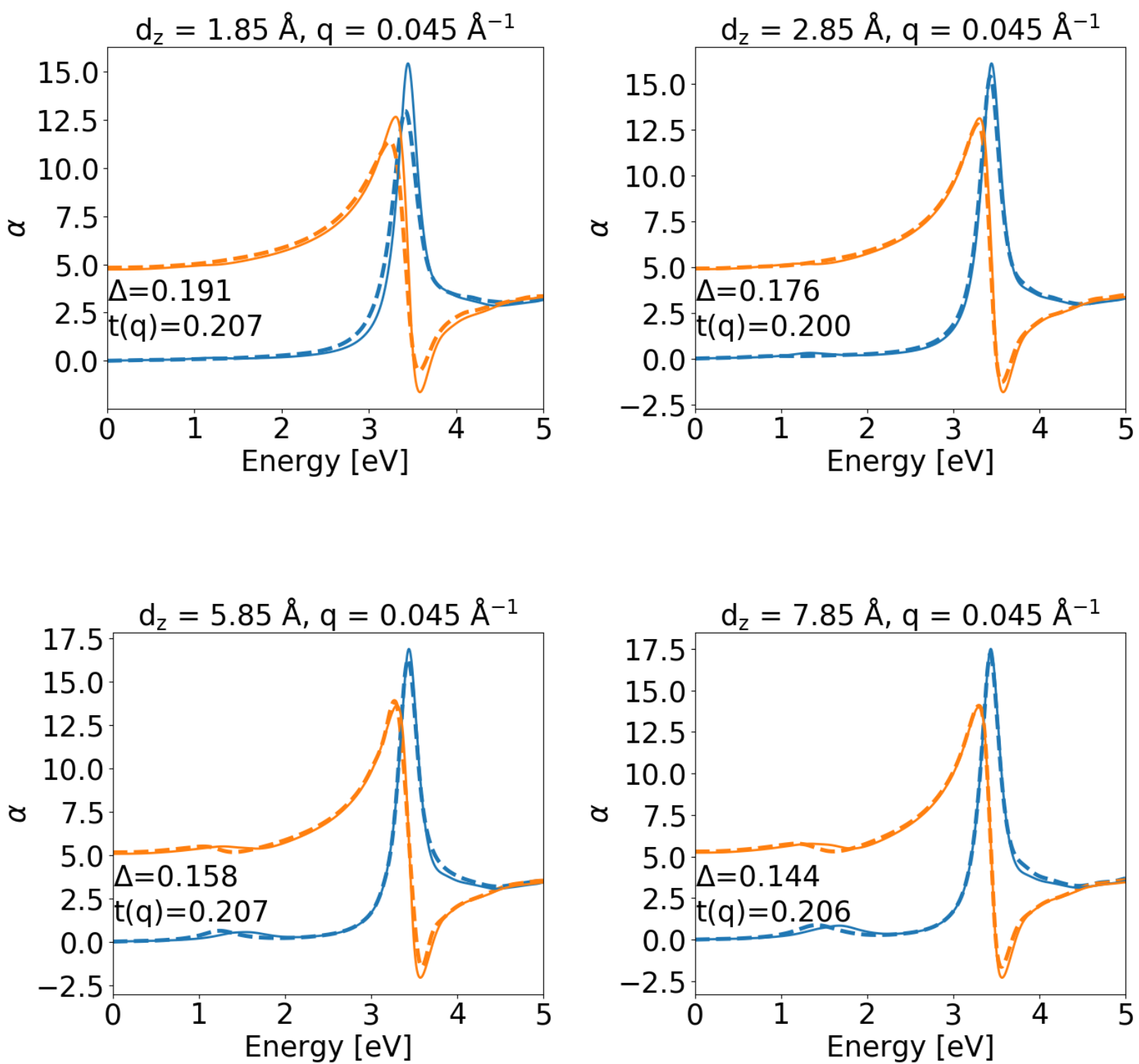

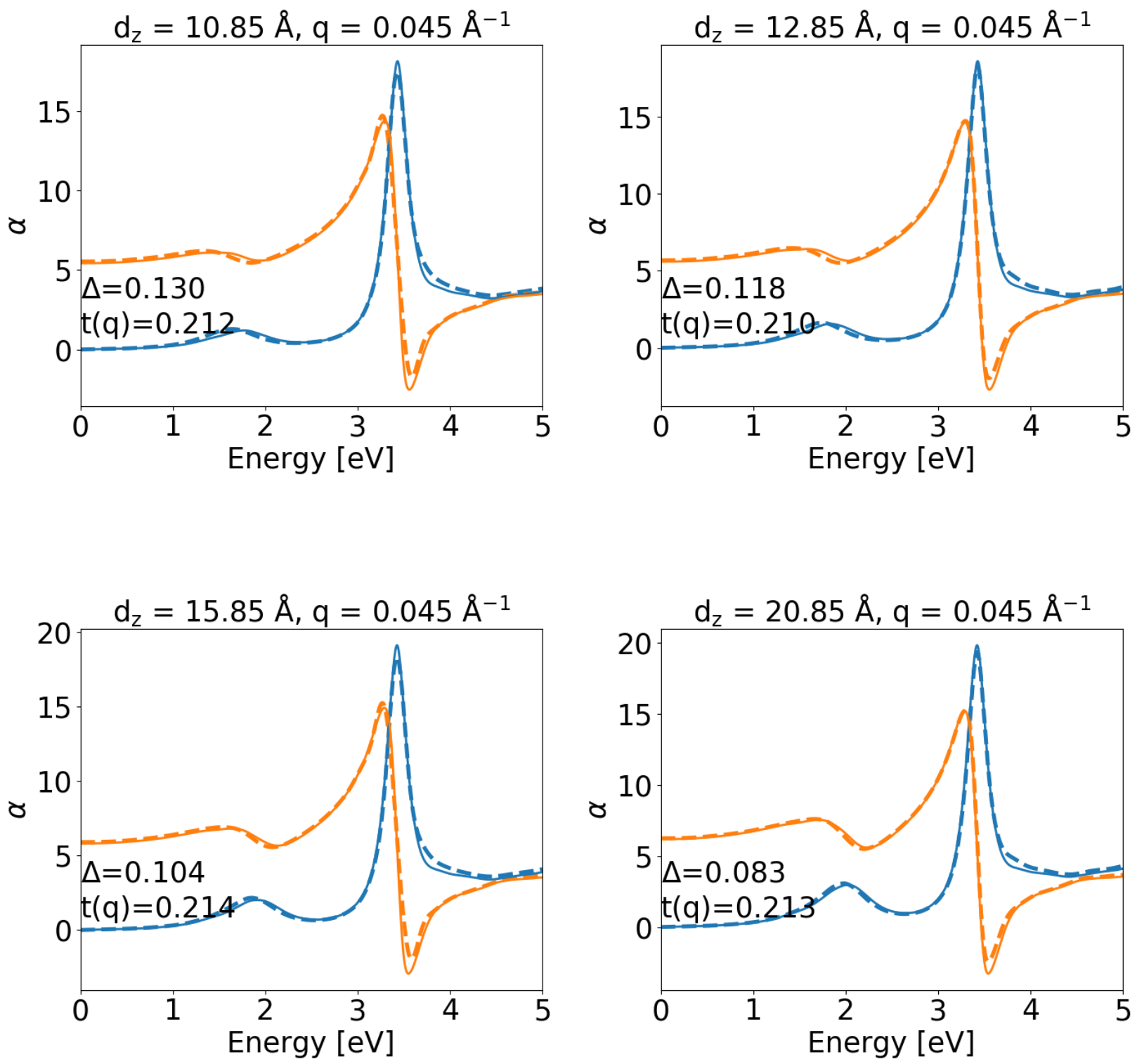

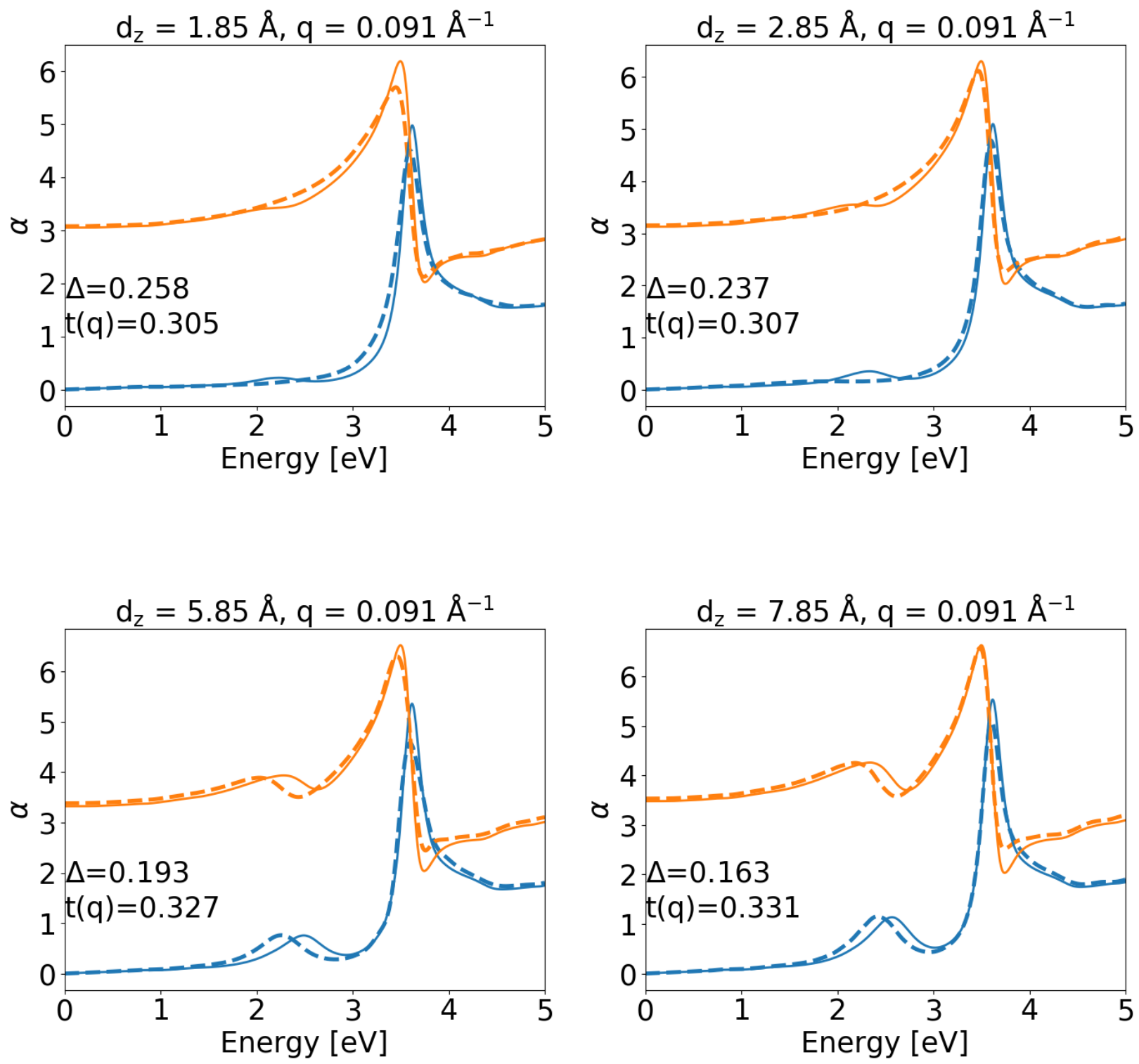

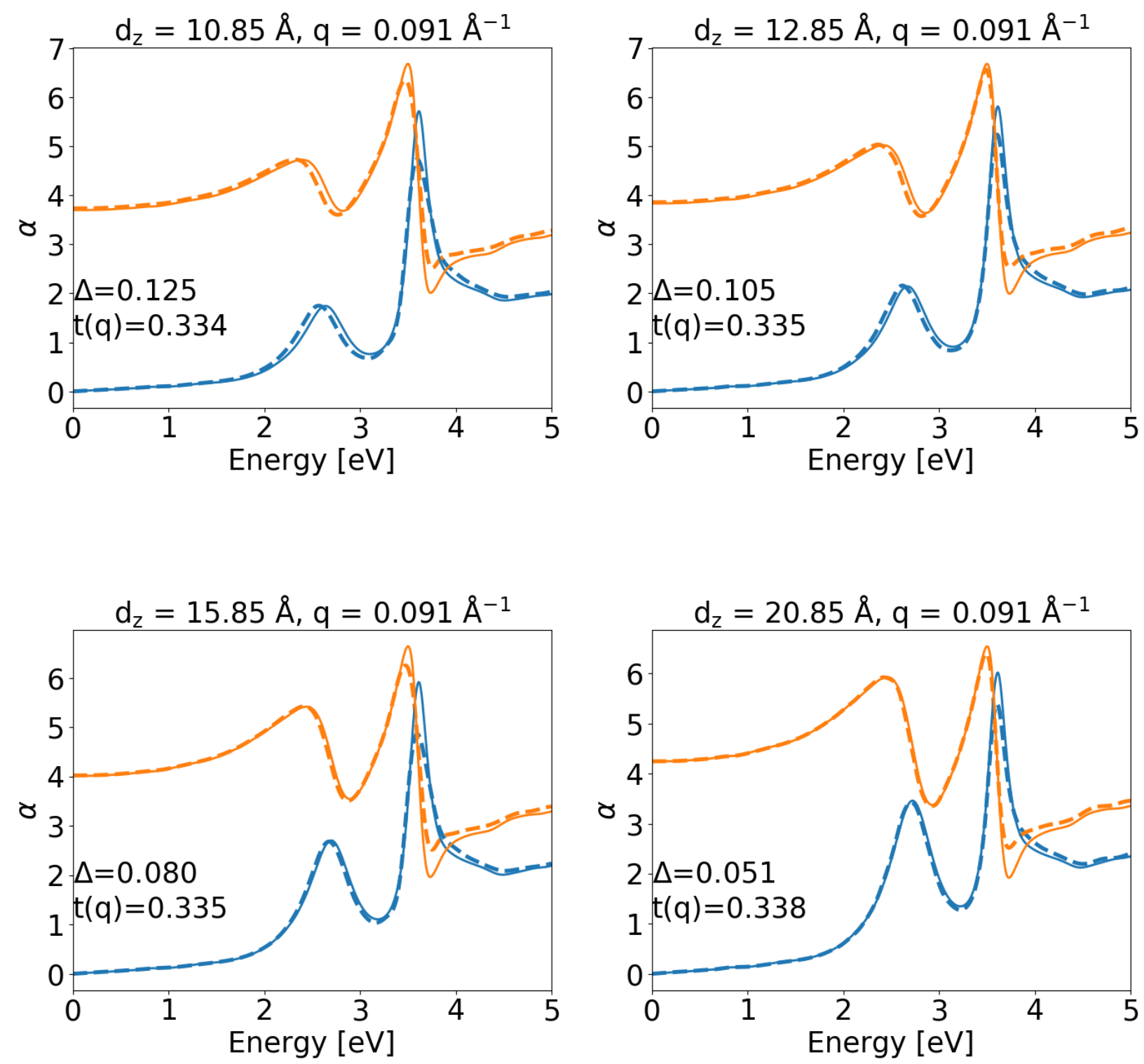

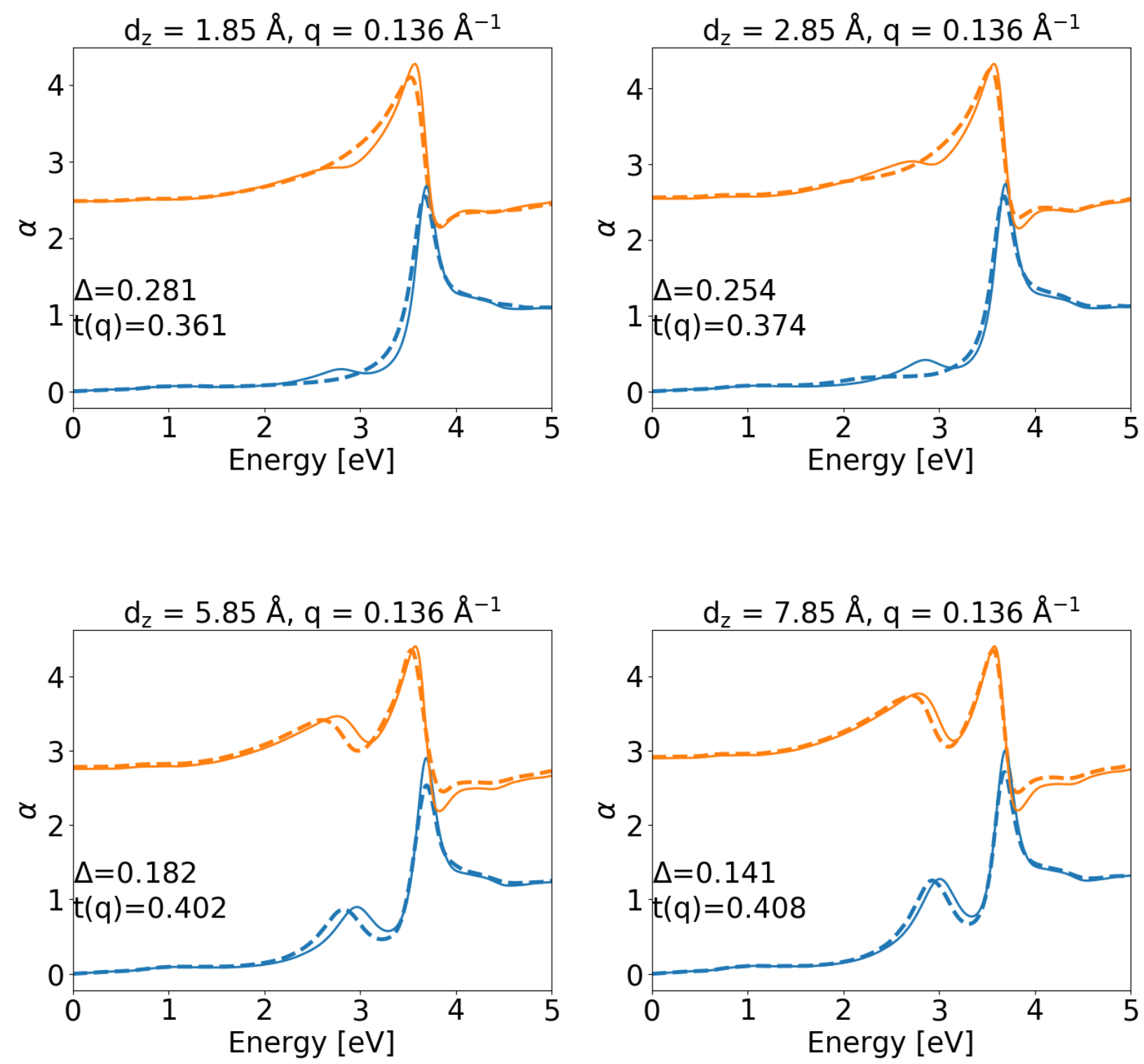

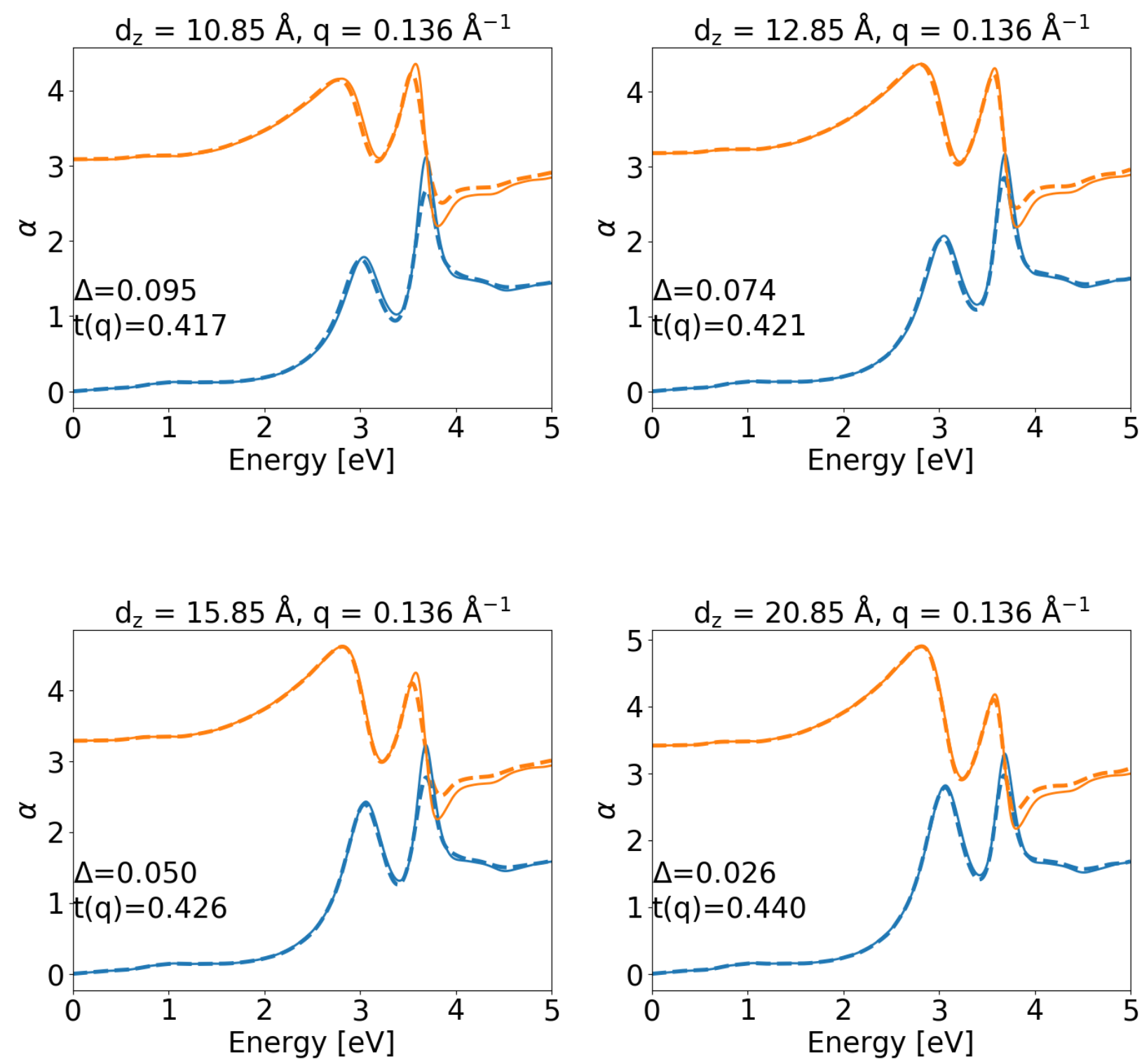

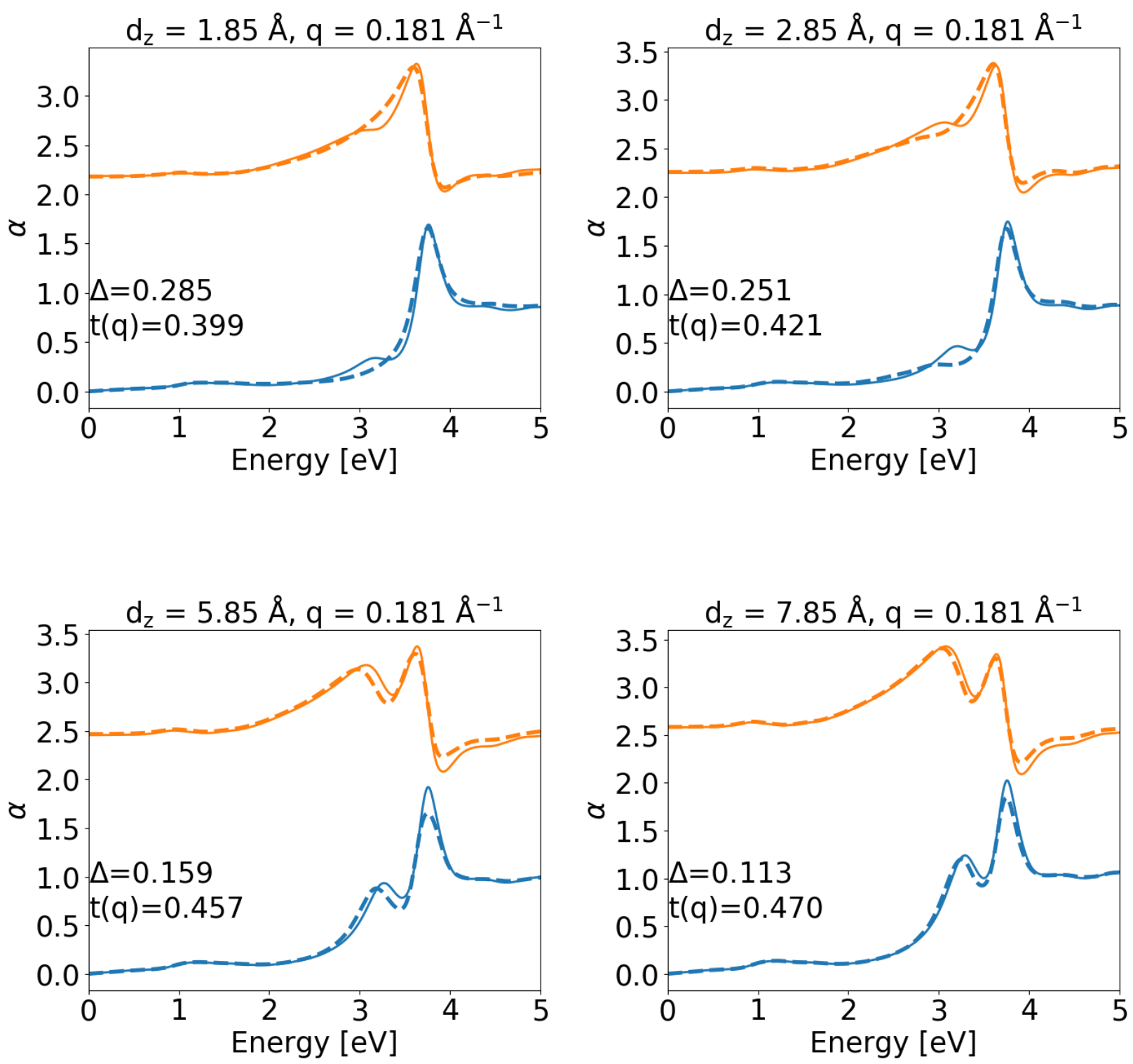

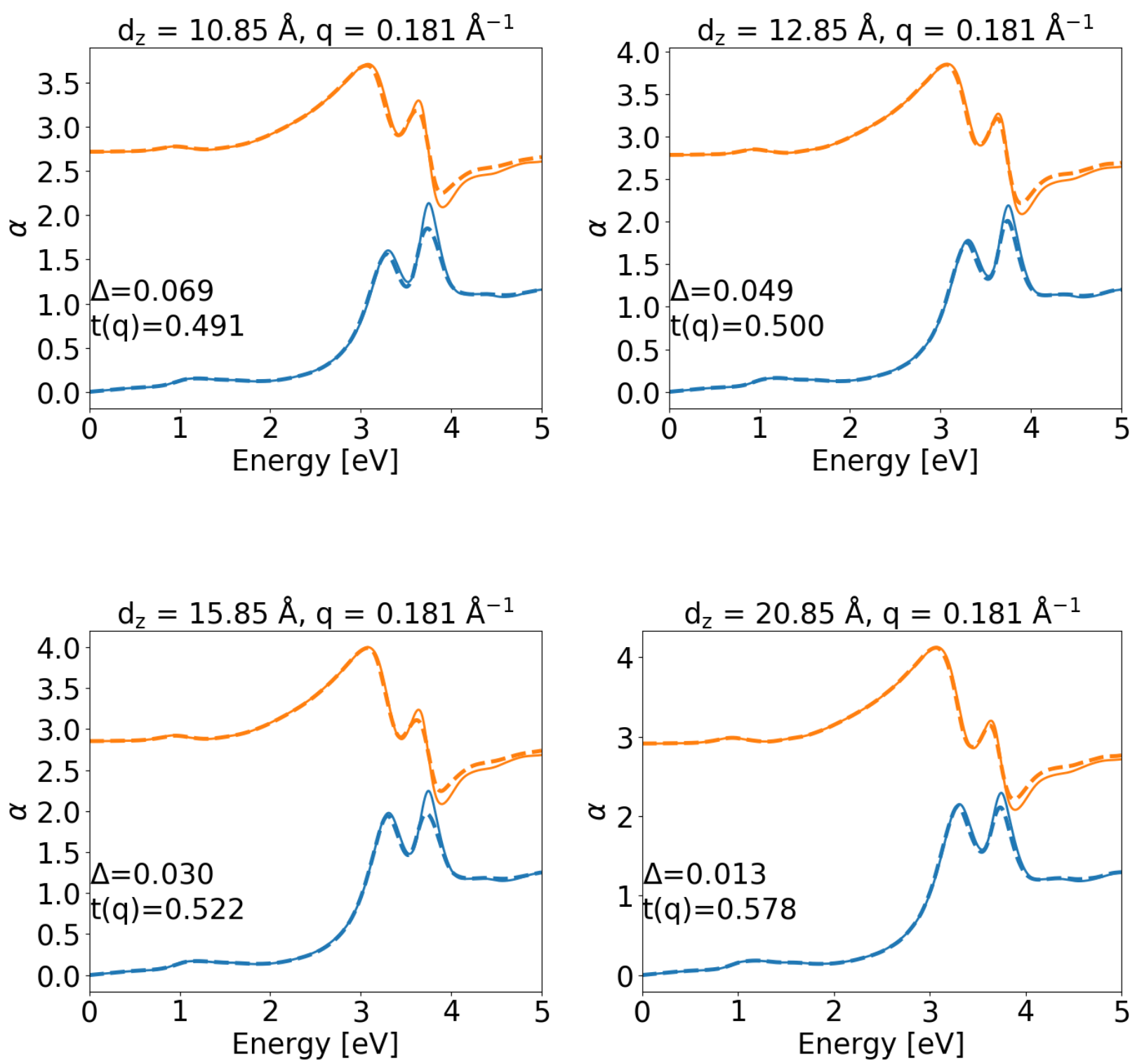

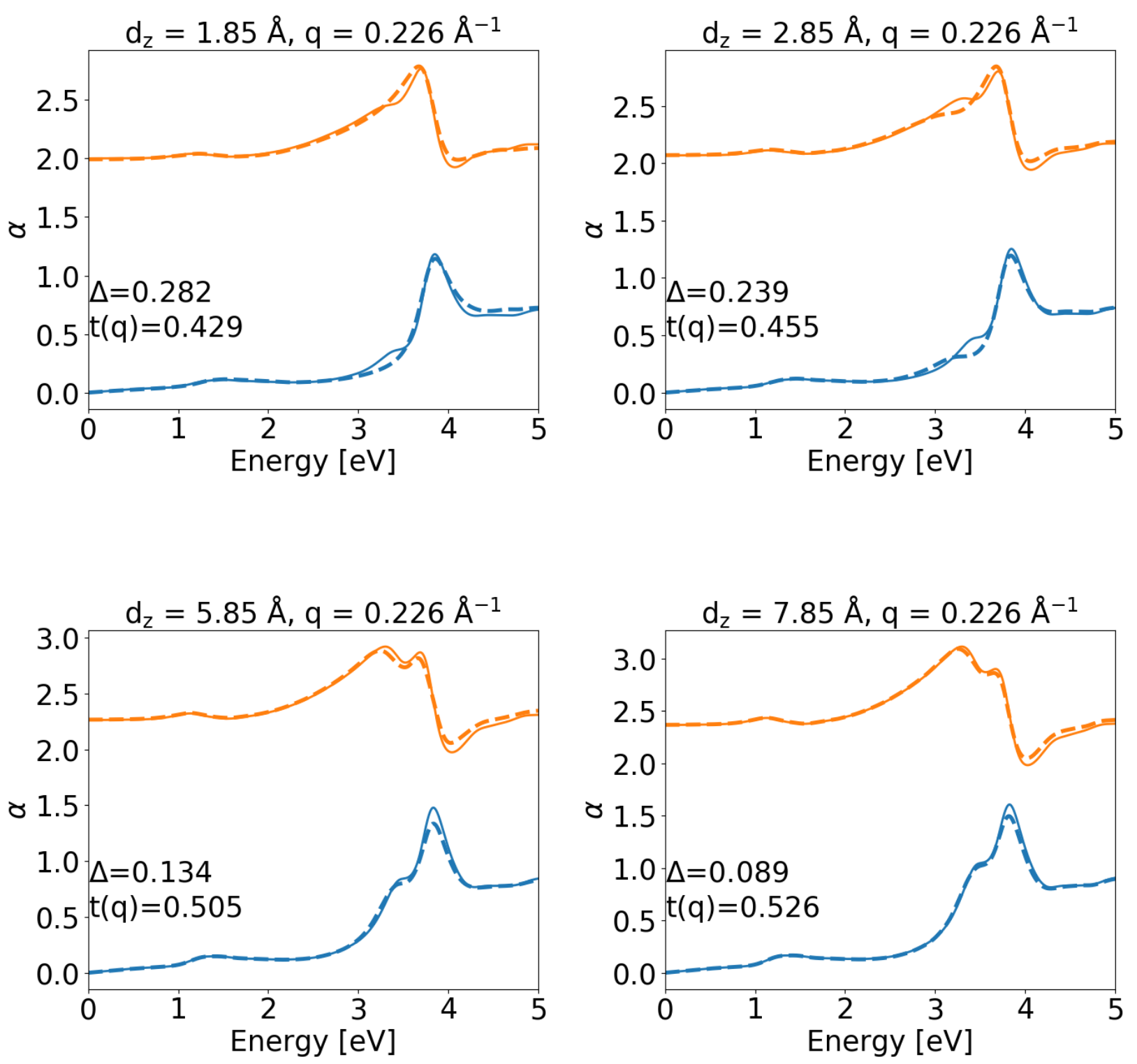

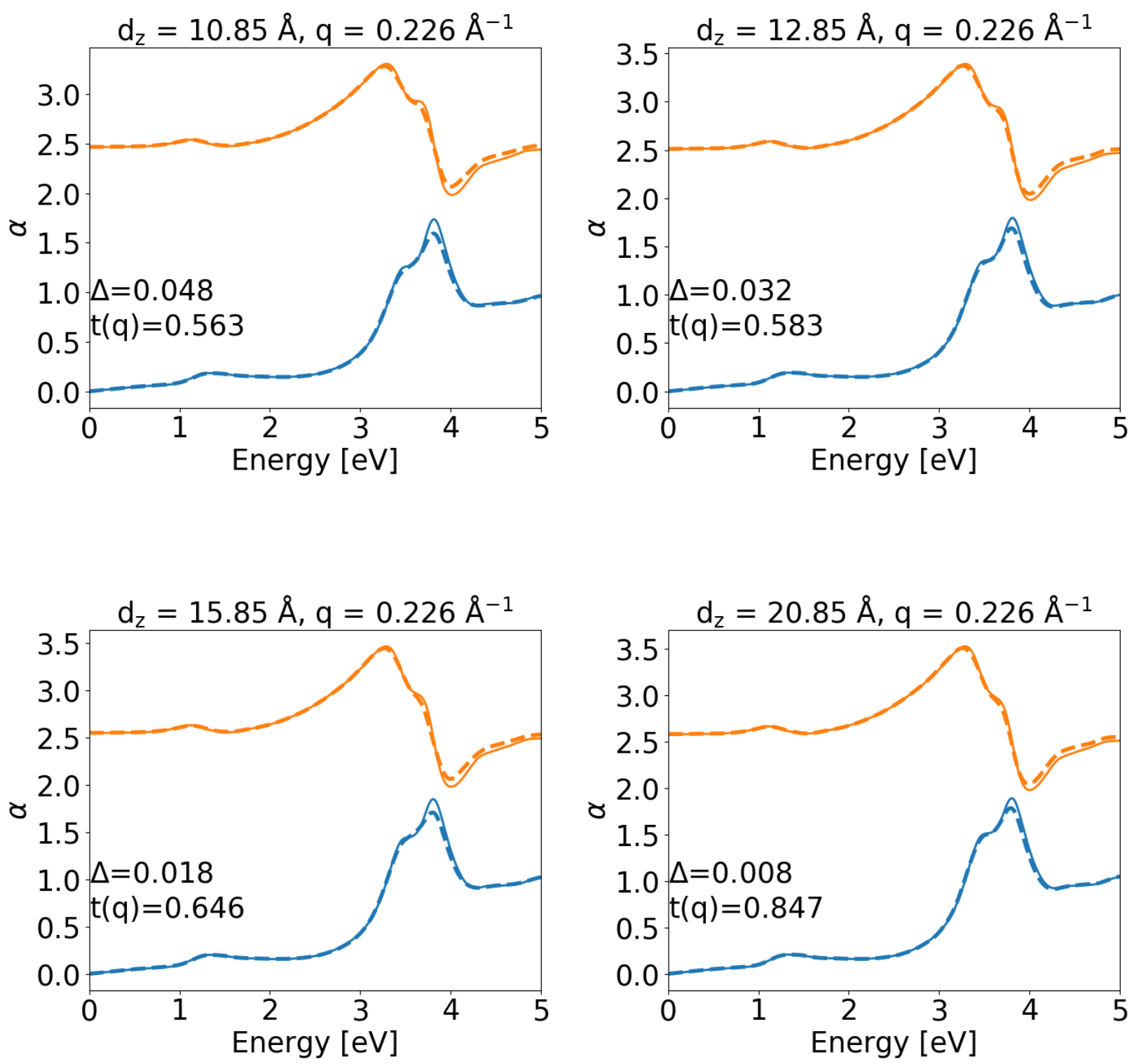


\section{Model robustness}

The sensitivity of the coupled polarizability (induced density) model response function to

fluctuations in $\mathrm{t}(\mathrm{q})$ for borophene/substrate. We determine that for $\mathbf{q d}>1$, the model is robust to fluctuations of $\pm 10 \%$. 


\subsection{Borophene/ $\operatorname{Ag}(111)$ : model sensitivity to $t(q)$}
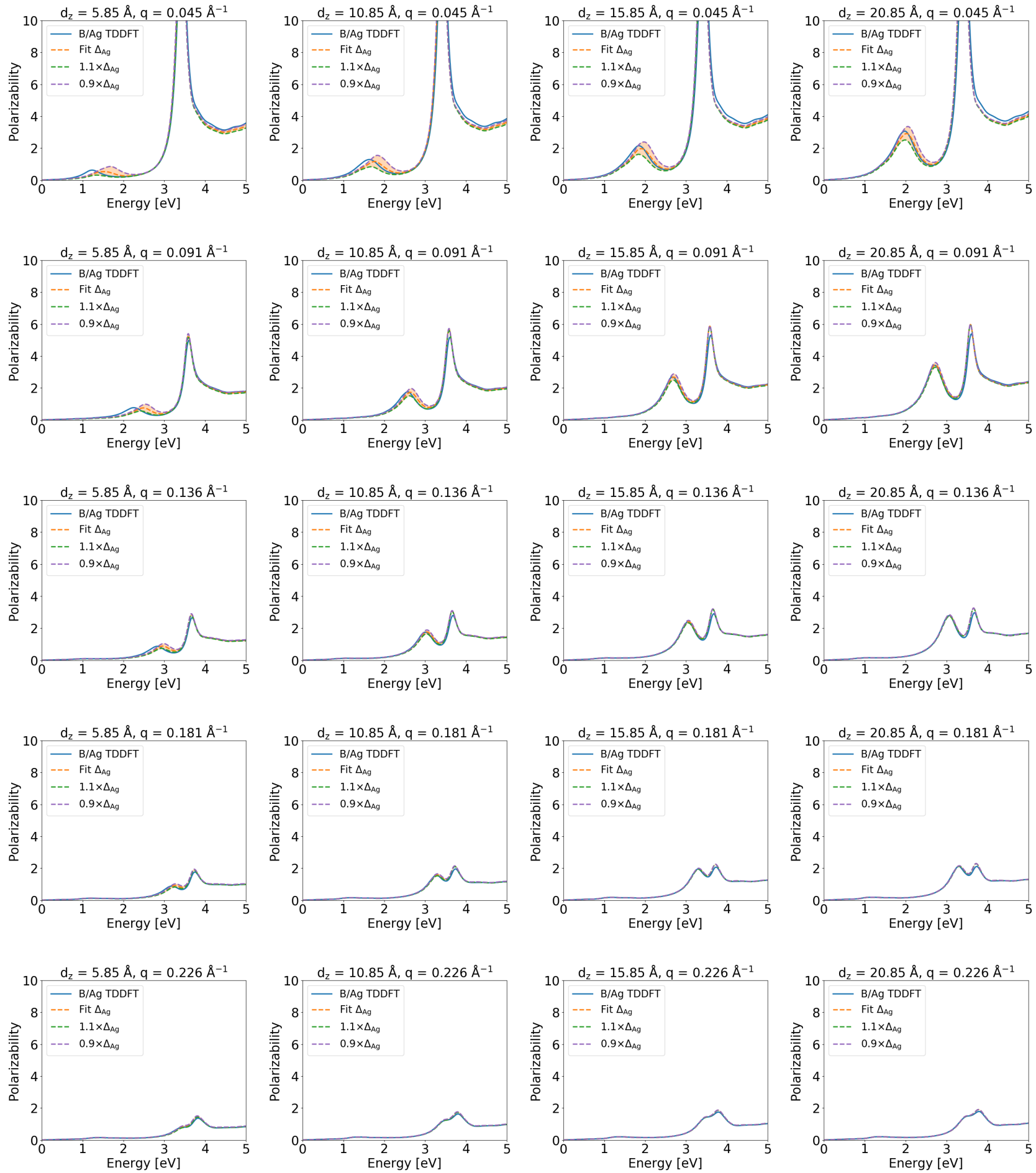

Figure 12: The sensitivity of the coupled mode model response function to fluctuations in $\mathrm{t}(\mathrm{q})$ for borophene/ $\mathrm{Ag}(111)$ compared with TDDFT calculations. The orange shaded regions around the orange dashed lines show the extent of the change in the modeled response if the coupling parameter is varied by $\pm 10 \%$ of its optimal value. 


\subsection{Borophene/Al(111): model sensitivity to $\mathrm{t}(\mathrm{q})$}
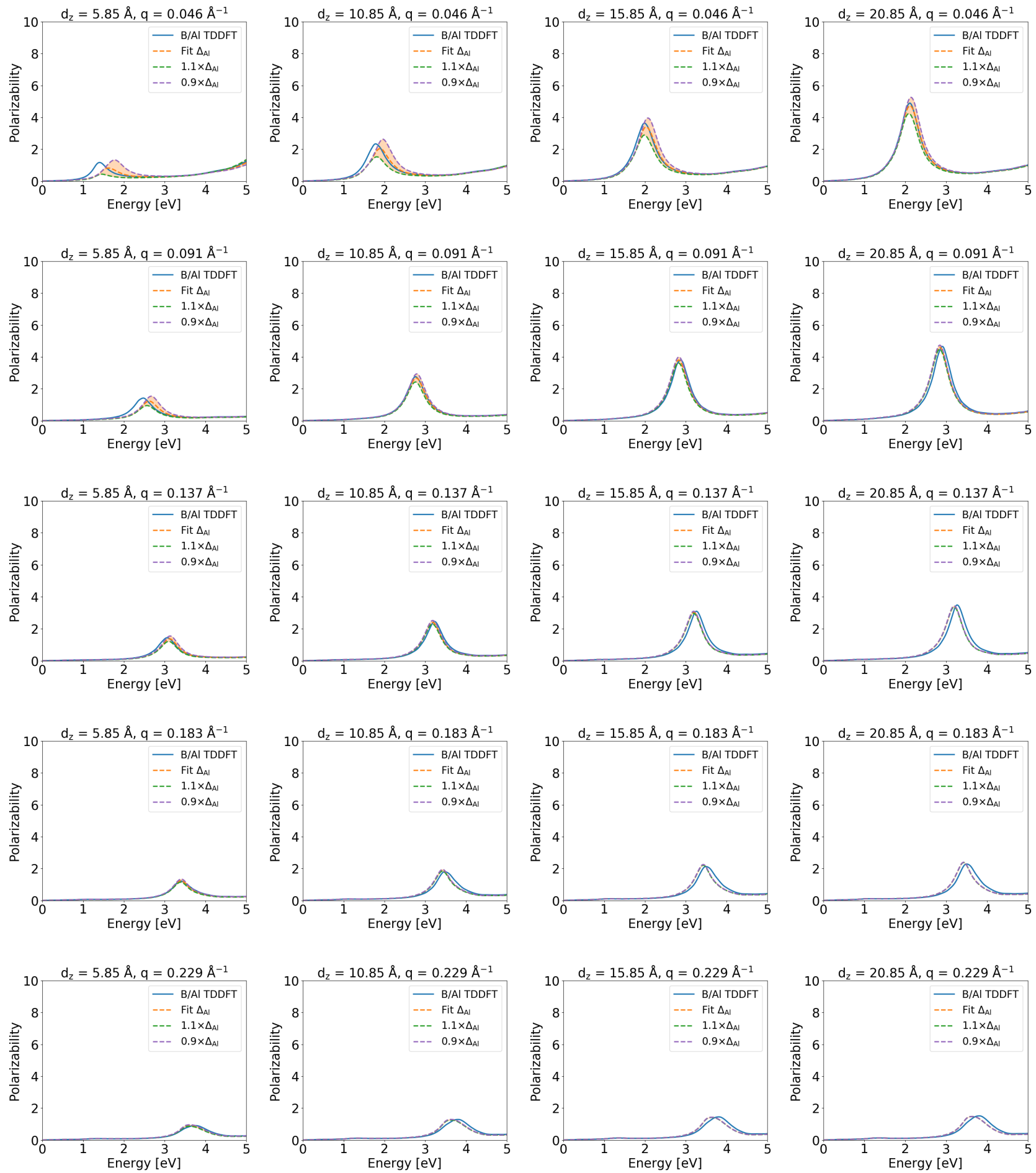

Figure 13: The sensitivity of the coupled mode model response function to fluctuations in $\mathrm{t}(\mathrm{q})$ for borophene/ $\mathrm{Al}(111)$ compared with TDDFT calculations. The orange shaded regions around the orange dashed lines show the extent of the change in the modeled response if the coupling parameter is varied by $\pm 10 \%$ of its optimal value. 


\subsection{Sensitivity to surface corrugation}
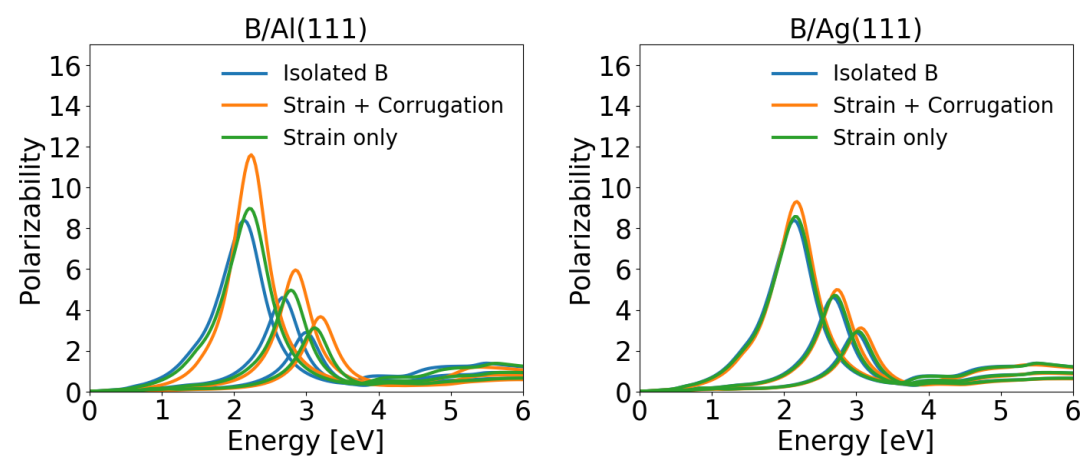

Figure 14: The effect of substrate-induced strain and corrugation on the imaginary part of borophene polarizability. The structure optimized at equilibrium, with both strain and corrugation included is shown in orange, while the green line shows the results when the borophene lattice constant is strained to be commensurate with the substrate. 


\section{Model transferability}

\subsection{Model-Predicted polarizability for borophene/Al(111) com- pared with fit}

The TDDFT-predicted polarizability of borophene/Al(111) along with our model as param-

eterized by the 1) borophene/ $\operatorname{Ag}(111)$ and 2) borophene/Al(111) response function. We determine that the fit to borophene/ $\mathrm{Ag}(111)$ reproduces the TDDFT spectra with accuracy suggesting that the mode is transferable to other metals. 

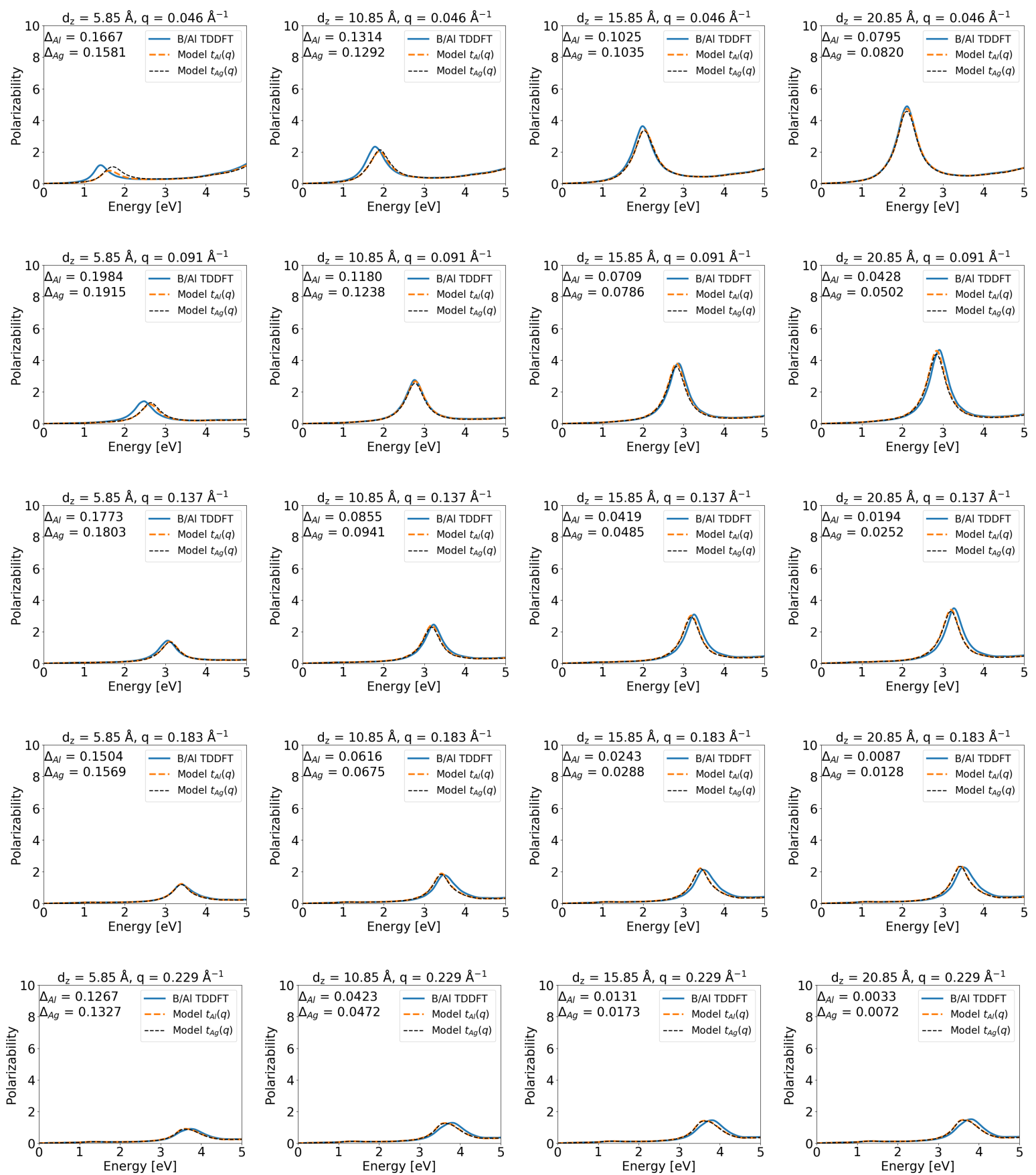

Figure 15: Predicted imaginary part of polarizability of borophene on $\mathrm{Al}(111)$ at varying distances above the image plane of $\mathrm{Al}(111)(5.85 \AA, 10.85 \AA, 15.85 \AA$ And $20.85 \AA)$, and at varying in-plane transferred momenta. The blue lines are TDDFT calculations, while the orange and black dashed lines are the coupled density model with parameters fit to borophene/ $\mathrm{Al}(111)$ and borophene/ $\mathrm{Ag}(111)$, respectively. The fit on $\mathrm{Ag}(111)$ was performed for the same distance from the image plane of $\mathrm{Ag}(111)$ and approximately the same q-vector; a discrepancy in $\mathrm{q}$ arising from the different lattice vectors of the $\mathrm{Ag}$ and $\mathrm{Al}$. The coupling parameter $\Delta$ are shown in the inset. For input to the model, the polarizability of the individual components (borophene and substrate) were those calculated for the optimized geometry at the interface. 


\subsection{Distance-dependence of the coupling parameter}

The coupling parameter $\Delta$, as a function of $-\mathrm{qd}_{\mathrm{Z}}$ on a $\log$ scale for borophene/ $\mathrm{Al}(111)$ and borophene/ $\mathrm{Ag}(111)$. Here, $\mathrm{d}_{\mathrm{z}}$ is the distance of borophene to the image plane of the metal. The linear relationship for both on a semilogy scale confirms the $\exp \left(-\mathrm{qd}_{\mathrm{z}}\right)$ behavior of $\Delta$.

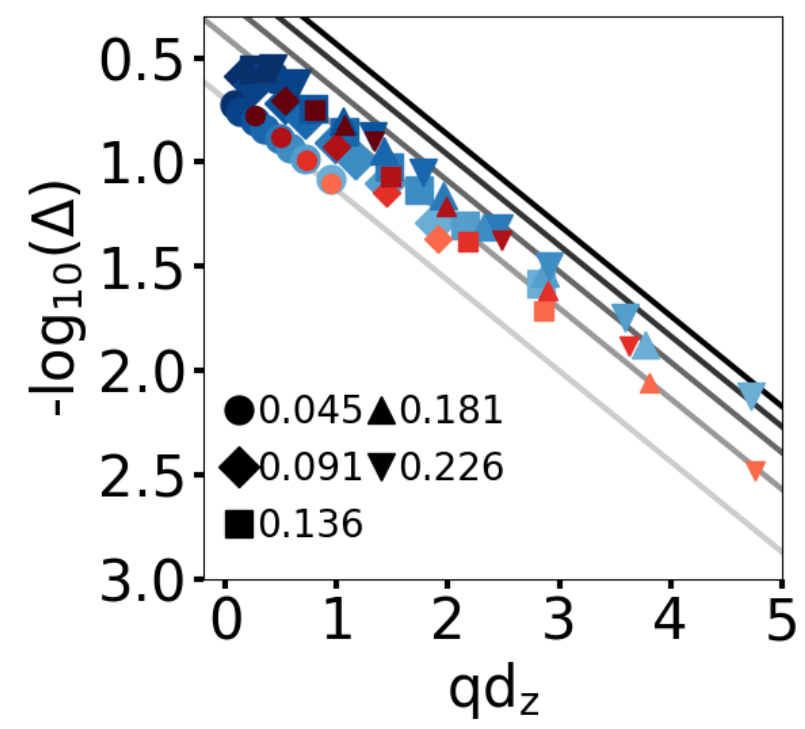

Figure 16: Comparison of $\Delta$ values between $\mathrm{B} / \mathrm{Al}(111)$ and $\mathrm{B} / \mathrm{Ag}(111)$. 

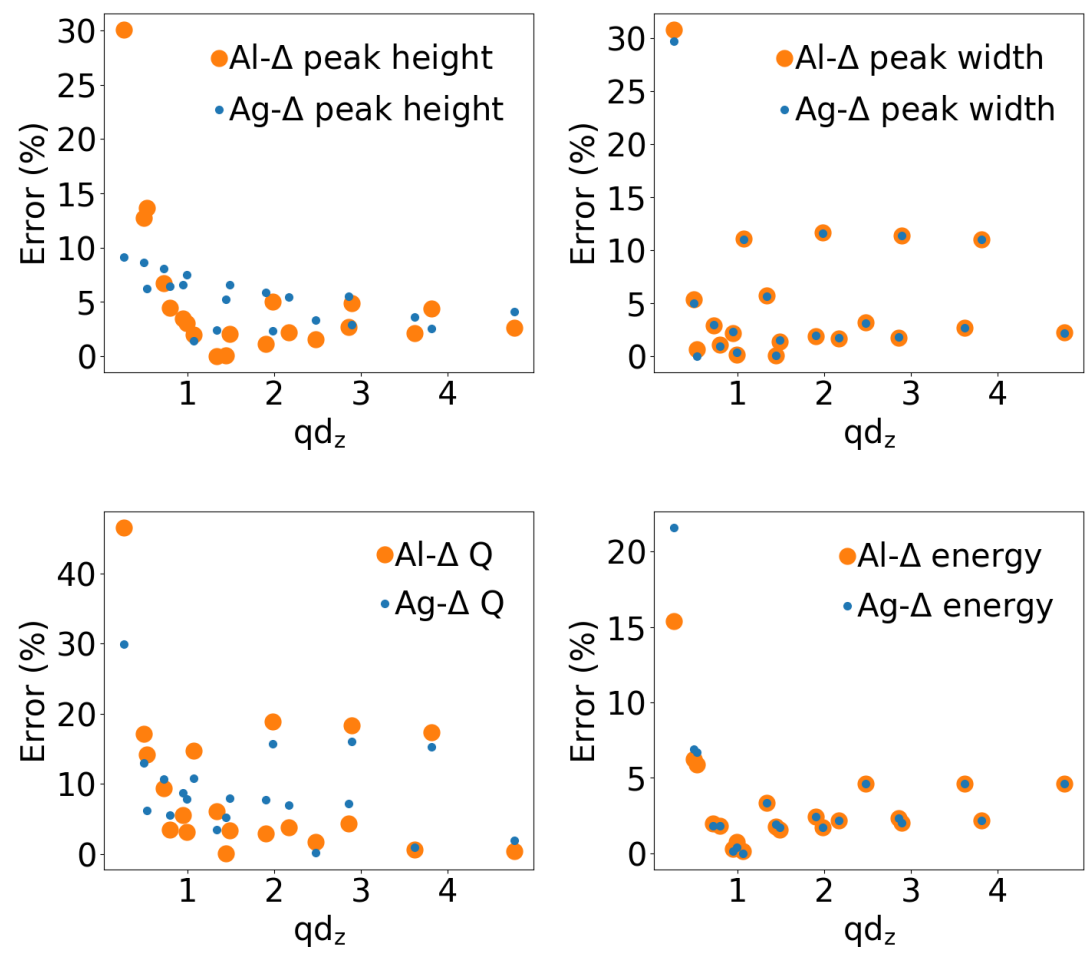

Figure 17: Error in model-predicted peak height, peak width, Q-factor and energy position when compared with TDDFT calculations of borophene/Al(111). The error for both borophene/Al(111)- and borophene/ $\mathrm{Ag}(111)$-parameterized models is shown and is calculated as the absolute value of the difference between TDDFT and the model. 


\section{Convergence}

We present representative calculations that show the convergence of linear response spectra as a function of the numerical parameters employed.

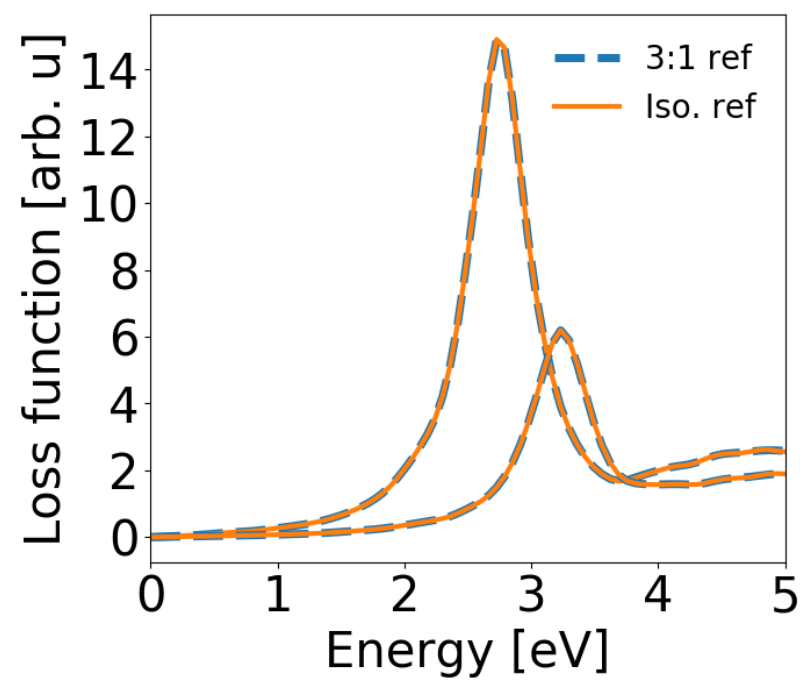

Figure 18: We used an anisotropic k-point density refinement for linear response calculations. The 3:1 anisotropic k-point refinement leads to converged loss spectra as compared to a full isotropic k-point density refinement.

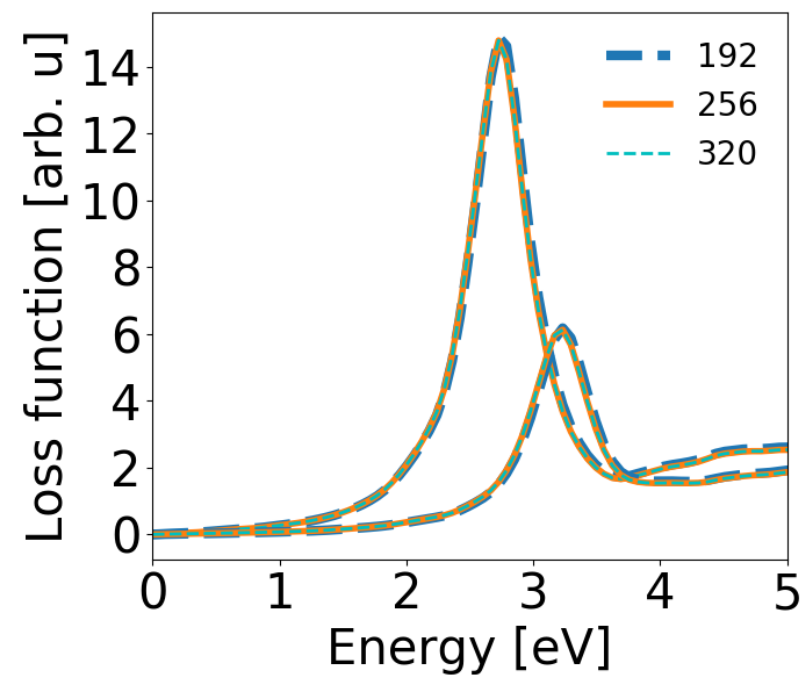

Figure 19: 192 bands were considered in the calculations of borophene/ $\mathrm{Ag}(111)$. Here we show that our loss spectra are converged in the energy range of interest: $<5 \mathrm{eV}$. 


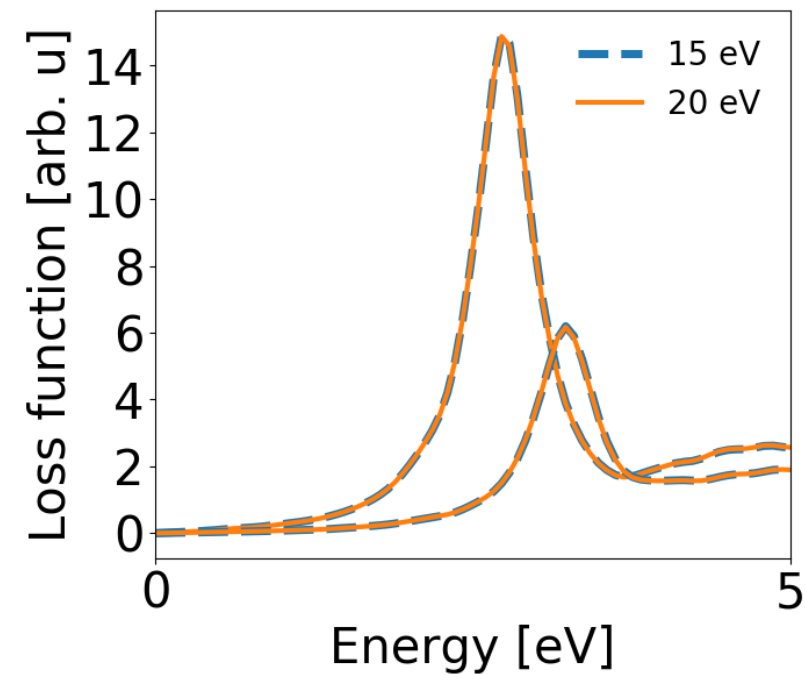

Figure 20: We considered linear response over a range of $15 \mathrm{eV}$, which is converged as a function of energy range.

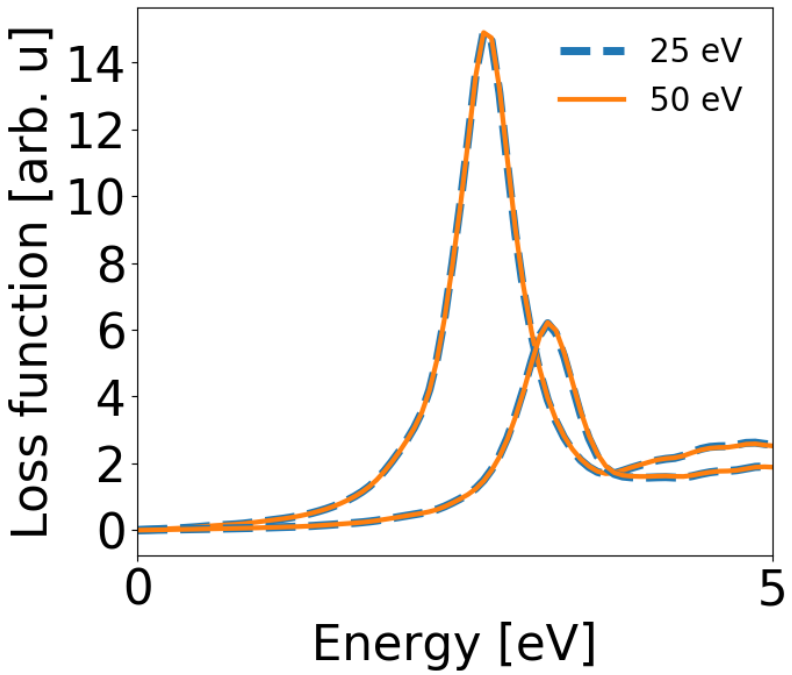

Figure 21: A local field effect planewave cutoff (dielectric matrix cutoff) of $25 \mathrm{eV}$ was sufficient to describe local fields for borophene on $\operatorname{Ag}(111)$.

\section{Scripts}

We include the following Python scripts with our supporting information, which were used to generate our data.

fsb_gllbsc_scf.py - Runs an SCF calculation on an optimized freestanding borophene 
geometry, converging occupied wavefunctions only.

fsb_gllbsc_finekpts.py - Runs a non-SCF calculations from the density of the fsb_gllbsc_scf.py script, converging empty states for a sum-over-states linear response calculation. Many more k-points are also chosen for this calculation to allow for smooth linear response spectra. fsb_gllbsc_eels.py - Runs a series of EELS/polarizability calculations over a range of transferred momenta q.

The silverb_ counterparts of these scripts run the above calculations for borophene/ $\mathrm{Ag}(111)$.

\section{References}

(1) Larsen, A. H. et al. J. Phys.: Condens. Matter 2017, 29, 273002.

(2) Jain, A.; Ong, S. P.; Hautier, G.; Chen, W.; Richards, W. D.; Dacek, S.; Cholia, S.; Gunter, D.; Skinner, D.; Ceder, G.; Persson, K. A. APL Materials 2013, 1, 011002.

(3) Perdew, J. P.; Burke, K.; Ernzerhof, M. Phys. Rev. Lett. 1996, 77, 3865-3868, Publisher: American Physical Society.

(4) Grimme, S.; Ehrlich, S.; Goerigk, L. Journal of Computational Chemistry 2011, 32, 1456-1465,_eprint: https://onlinelibrary.wiley.com/doi/pdf/10.1002/jcc.21759.

(5) Ceperley, D. M.; Alder, B. J. Phys. Rev. Lett. 1980, 45, 566-569.

(6) Perdew, J. P.; Zunger, A. Phys. Rev. B 1981, 23, 5048-5079.

(7) Kresse, G.; Furthmüller, J. Phys. Rev. B 1996, 54, 11169-11186.

(8) Kresse, G.; Furthmüller, J. Computational Materials Science 1996, 6, 15-50.

(9) Blöchl, P. E. Phys. Rev. B 1994, 50, 17953-17979.

(10) Kresse, G.; Joubert, D. Phys. Rev. B 1999, 59, 1758-1775.

(11) Methfessel, M.; Paxton, A. T. Phys. Rev. B 1989, 40, 3616-3621. 
(12) Liu, X.; Wang, L.; Li, S.; Rahn, M. S.; Yakobson, B. I.; Hersam, M. C. Nat. Commun. 2019, 10, 1642 .

(13) Kuisma, M.; Ojanen, J.; Enkovaara, J.; Rantala, T. T. Phys. Rev. B 2010, 82, 115106.

(14) Yan, J.; Mortensen, J. J.; Jacobsen, K. W.; Thygesen, K. S. Phys. Rev. B 2011, 83, 245122.

(15) Mortensen, J. J.; Hansen, L. B.; Jacobsen, K. W. Phys. Rev. B 2005, 71, 035109.

(16) Enkovaara, J. et al. J. Phys.: Condens. Matter 2010, 22, 253202.

(17) Branch, M. A.; Coleman, T. F.; Li, Y. SIAM Journal on Scientific Computing 1999, 21, 1-23.

(18) Triggs, B.; McLauchlan, P. F.; Hartley, R. I.; Fitzgibbon, A. W. Bundle Adjustment — A Modern Synthesis. Vision Algorithms: Theory and Practice. Berlin, Heidelberg, $2000 ;$ pp 298-372.

(19) Zwanzig, R. Physical Review 1961, 124, 983-992.

(20) Mori, H. Progress of Theoretical Physics 1965, 34, 399-416.

(21) Darancet, P.; Olevano, V.; Mayou, D. Phys. Rev. B 2010, 81, 155422. 